\title{
The influence of thermal boundary conditions on turbulent forced convection pipe flow at two Prandtl numbers
}

\author{
Steffen Straub ${ }^{\mathrm{a}, *}$, Pourya Forooghi $^{\mathrm{a}}$, Luca Marocco ${ }^{\mathrm{b}}$, Thomas Wetzel ${ }^{\mathrm{c}}$, \\ Ricardo Vinuesa $^{\mathrm{d}}$, Philipp Schlatter ${ }^{\mathrm{d}}$, Bettina Frohnapfel ${ }^{\mathrm{a}}$ \\ ${ }^{a}$ Institute of Fluid Mechanics, Karlsruhe Institute of Technology, 76131 Karlsruhe, \\ Germany \\ ${ }^{b}$ Dipartimento di Energia, Politecnico di Milano, 20156 Milano, Italy \\ ${ }^{c}$ Institute of Thermal Process Engineering, Karlsruhe Institute of Technology, 76131 \\ Karlsruhe, Germany \\ ${ }^{d}$ Linné FLOW Centre, KTH Mechanics, SE-100 44 Stockholm, Sweden
}

\begin{abstract}
Different types of thermal boundary conditions are conceivable in numerical simulations of convective heat transfer problems. Isoflux, isothermal and a mixedtype boundary condition are compared by means of direct numerical simulations (for the lowest Reynolds number) and well-resolved large-eddy simulations of a turbulent forced convection pipe flow over a range of bulk Reynolds numbers from $R e_{b}=5300$ to $R e_{b}=37700$, at two Prandtl numbers, i.e. $\operatorname{Pr}=0.71$ and $\operatorname{Pr}=0.025$. It is found that, while for $\operatorname{Pr}=0.71$ the Nusselt number is hardly affected by the type of thermal boundary condition, for $\operatorname{Pr}=0.025$ the isothermal boundary condition yields $\approx 20 \%$ lower Nusselt numbers compared to isoflux and mixed-type over the whole range of Reynolds numbers. A decomposition of the Nusselt number is derived. In particular, we decompose it into four contributions: laminar, radial and streamwise turbulent heat flux as well as a contribution due to the turbulent velocity field. For $\operatorname{Pr}=0.71$ the contribution due to the radial turbulent heat flux is dominant, whereas for $\operatorname{Pr}=0.025$ the contribution due to the turbulent velocity field is dominant. Only at a moderately high Reynolds number, such as $R e_{b}=37700$, both turbulent con-
\end{abstract}

\footnotetext{
* Corresponding author

Email address: steffen.straub@kit.edu (Steffen Straub)
} 
tributions are of similar magnitude. A comparison of first- and second-order thermal statistics between the different types of thermal boundary conditions shows that the statistics are not only influenced in the near-wall region but also in the core region of the flow. Power spectral densities illustrate large thermal structures in low-Prandtl-number fluids as well as thermal structures located right at the wall, only present for the isoflux boundary condition.

A database including the first- and second-order statistics together with individual contributions to the budget equations of the temperature variance and turbulent heat fluxes is hosted in the open access repository KITopen (DOI:10.5445/IR/1000096346).

Keywords: thermal boundary conditions, low Prandtl number, forced convection, pipe, turbulence

\section{Nomenclature}

\section{Latin Symbols}

a Exponential decay rate for isothermal (IT) boundary condition

A Cross section of the pipe

5 c $c_{p} \quad$ Specific heat capacity at constant pressure

$D \quad$ Pipe diameter

E Power spectral density

F Forcing term to drive the flow

$\mathscr{H} \quad$ High-pass spectral filter for relaxation-term

${ }_{10} h \quad$ Channel half-height

$k_{z} \quad$ Streamwise wave number

$L_{z} \quad$ Pipe length

$N u \quad$ Nusselt number $\left(q_{w} D\right) /\left(\left(\left\langle T_{w}\right\rangle^{t}-T_{b}\right) \lambda\right)$

$N u_{H F}$ Nusselt number contribution due to turbulent heat flux

${ }_{15} \quad N u_{L} \quad$ Laminar Nusselt number contribution

$N u_{R S S}$ Nusselt number contribution due to the turbulent velocity field

$p \quad$ Pressure 


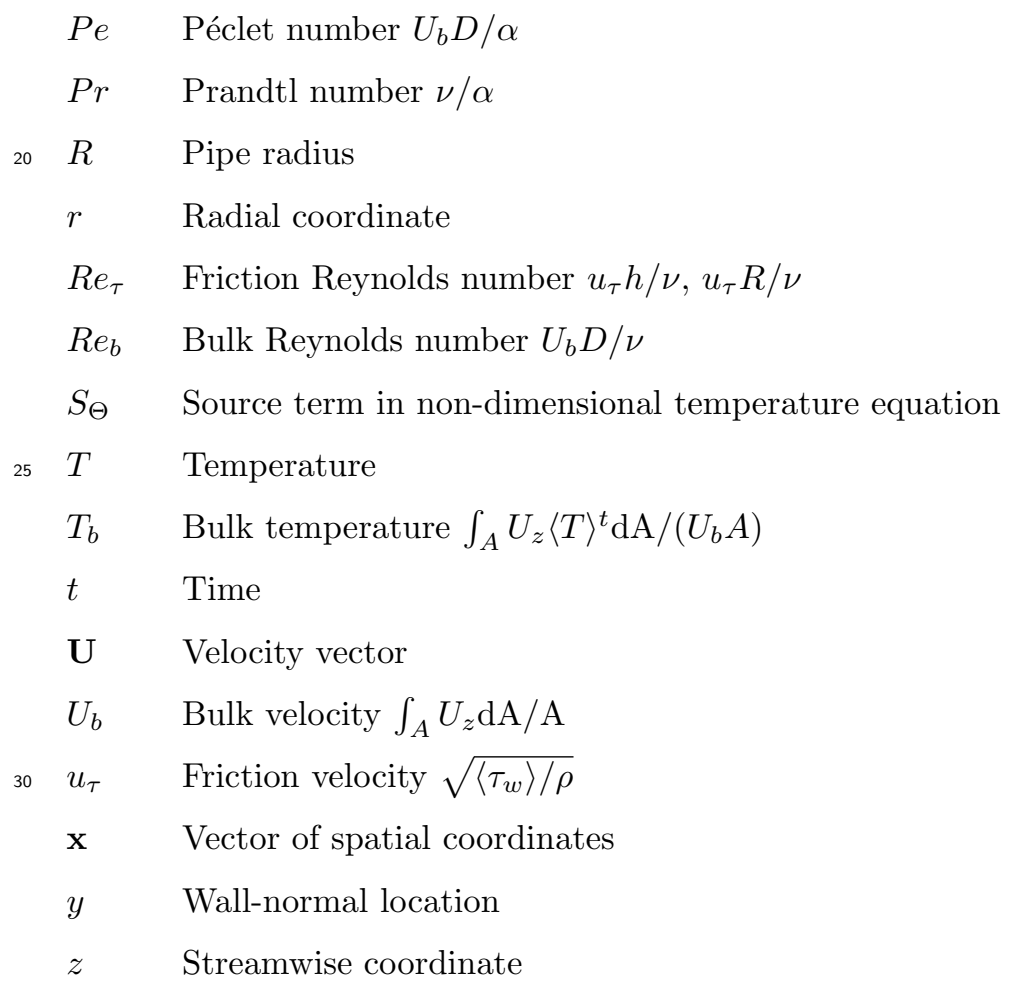

${ }_{35} \quad \alpha \quad$ Thermal diffusivity $\lambda /\left(\rho c_{p}\right)$

$\chi \quad$ Model parameter for relaxation-term

$\lambda \quad$ Thermal conductivity

$\lambda_{z} \quad$ Streamwise wavelength

$\nu \quad$ Kinematic viscosity

40 $\Phi \quad$ Fractional flow rate

$\Phi_{T} \quad$ Fractional flow rate of the turbulent velocity field

$\varphi \quad$ Azimuthal coordinate

$\rho \quad$ Density

$\tau_{w} \quad$ Wall-shear stress

${ }_{45} \Theta \quad$ Non-dimensional temperature $\left(\left\langle T_{w}\right\rangle^{t}-T\right) /\left\langle q_{w}\right\rangle^{t} \rho c_{p} U_{b}$

$\vartheta \quad$ Fluctuating part of non-dimensional temperature

\section{Superscipts}




$\begin{array}{ll}* & \text { Complex conjugate } \\ + & \text { Scaling in viscous units } \\ t & \text { Time (average) } \\ \text { Subscripts } \\ r & \text { Radial component } \\ w & \text { Wall (temperature, heat flux) } \\ z & \text { Streamwise component }\end{array}$

55 Abbreviations

CSP Concentrated solar power

DNS Direct numerical simulation

IF Isoflux

IT Isothermal

60 LES Large-eddy simulation

MBC Mixed-type boundary condition

PSD Power spectral density

RSS Reynolds shear stress

TBC Thermal boundary condition

65 1. Introduction

Since the first direct numerical simulation (DNS) of a turbulent channel flow by Kim et al. 1], DNS has become an important tool to study the physics of wall bounded turbulent flow and turbulent forced convection, where additionally to the Navier-Stokes equations an advection-diffusion equation is solved for temperature, treated as a passive scalar. Kasagi et al. 2] performed simulations with a prescribed time-averaged wall heat flux boundary condition in order to mimic the realistic thermal boundary condition (TBC) governed by a conjugate heat transfer problem. This type of boundary condition is sometimes referred to as mixed or mixed-type boundary condition (MBC) because the wall is as75 sumed to be locally isothermal, i.e. temperature fluctuations vanish at the wall, 
whereas the averaged temperature increases linearly in the streamwise direction similar to isoflux boundary conditions. The MBC has been used to study the influence of the Reynolds and Prandtl numbers in the channel by Kawamura et al. 3] up to a Reynolds number of $R e_{\tau}=u_{\tau} h / \nu=395$. Abe et al. [4 presented results for Reynolds numbers up to $R e_{\tau}=1020$ using the MBC in a channel flow with $\operatorname{Pr}=0.025$ and $\operatorname{Pr}=0.71$.

Previous studies on the effect of the type of TBC compare constant temperature and constant wall heat flux in a thermal boundary layer [5], and they also propose solving a conjugate heat transfer problem together with the limiting cases of fluctuating and non-fluctuating TBCs in fully developed channel flow simulations [6]. The first study comparing the three types of TBCs in a pipe geometry discussed in the following sections, namely MBC, isoflux (IF, where the wall heat flux is constant in time and space) and isothermal (IT, where the wall temperature is constant in time and space) was carried out by 90 Piller [7. He considered a turbulent pipe flow at a friction Reynolds number of $R e_{\tau}=u_{\tau} R / \nu=180$ and a Prandtl number of $\operatorname{Pr}=\nu / \alpha=0.71$ and concluded that MBC differs from IF only within the conduction sublayer. It is not known whether this conclusion also holds for low-Prandtl-number fluids or higher Reynolds numbers. Flageul et al. [8] presented a comparison of MBC, IF, 95 Robin boundary condition (linear combination of prescribed wall temperature and wall heat flux) and a conjugate heat transfer condition for turbulent channel flow at $R e_{\tau}=150$ and $\operatorname{Pr}=0.71$. They pointed out the importance of accurate predictions of temperature fluctuations at the wall, being sensitive to the type of TBC, as those cause fluctuating thermal stresses which can be a highly relevant parameter for industrial applications. Pirozzoli et al. [9] study passive scalar transport in channel flow at high Reynolds numbers up to $R e_{\tau}=4088$ for $\operatorname{Pr}=\{0.2,0.71,1\}$. They apply a source term in the temperature equation so that the integral of the non-dimensional temperature remains strictly constant in time as well as a prescribed temperature difference between upper and lower wall of the channel. By analyzing the effects of the employed source term on the thermal statistics, they find that the two different types of applied TBCs yield 
differences mainly in the core region of the flow. Alcántara-Ávila et al. [10] performed DNSs in channel flow up to $R e_{\tau}=2000$ for Prandtl numbers in the range of $\operatorname{Pr}=0.007$ to $\operatorname{Pr}=0.71$ (liquid metals - air). Comparing their results, using the MBC, to the spatially uniform forcing of Pirozzoli et al. 9], they find only small deviations with MBC resulting in slightly increased mean temperature, temperature variance and turbulent heat fluxes. Besides simulations conducted in the channel, the influence of the thermal boundary condition has also been studied for spatially developing turbulent boundary layer by Li et al. 11 in the context of passive scalar transport. Prandtl numbers $\operatorname{Pr}=\{0.2,0.71,2\}$ are considered at a Reynolds number based on free-stream velocity and momentum thickness of $R e_{\theta}=830$. They compare isoscalar (i.e. IT) and isoflux boundary conditions and find no effect on the mean thermal profiles but on the temperature variance. They also report one-dimensional premultiplied energy spectra indicating the differences between both boundary conditions in the near-wall region.

The purpose of the present study is to expand the findings by Piller [7] to higher Reynolds numbers and to lower Prandtl numbers. Low Prandtl numbers are characteristic for liquid metals, which have been proposed as heat transfer fluids in concentrated solar power (CSP) plants by Pacio et al. [12, where accurate predictions of the local wall temperature in the receiver are essential for an efficient design. Therefore, a profound knowledge about the influence of the type of $\mathrm{TBC}$ is required in order to select the most appropriate one, depending on the problem to solve. Furthermore, the present database can be useful to develop improved computational models for CSP plants, as in the work by Vinuesa et al. 13.

\section{Governing Equations and Numerical Method}

Numerical simulations are performed with the high-order spectral element code Nek5000 [14. The applied spectral element method combines the geo- 
methods by decomposing the domain into elements and solving for the unknowns by means of a weighted residual technique (Galerkin formulation) using high order orthogonal polynomials as basis functions [15, 16.

The numerical domain is a straight pipe of length $12.5 \mathrm{D}$ with periodic boundary conditions. The set of partial differential equations to be solved are the Navier-Stokes equations for an incompressible Newtonian fluid with constant thermophysical properties together with an advection-diffusion equation for temperature:

$$
\begin{aligned}
& \frac{\mathrm{D} \tilde{\mathbf{U}}}{\mathrm{D} \tilde{t}}=-\tilde{\nabla} \tilde{p}+\frac{1}{R e_{b}} \tilde{\nabla}^{2} \tilde{\mathbf{U}}+\tilde{\mathbf{F}}, \quad \tilde{\nabla} \cdot \tilde{\mathbf{U}}=0 \\
& \frac{\mathrm{D} \Theta}{\mathrm{D} \tilde{t}}=\frac{1}{P e} \tilde{\nabla}^{2} \Theta+S_{\Theta}
\end{aligned}
$$

The non-dimensional temperature $\Theta$ is treated as a passive scalar and viscous dissipation is neglected in the energy equation [17. Instantaneous velocity $\mathbf{U}$, time $t$, spatial coordinates $\mathbf{x}$, pressure $p$ and the forcing term $\mathbf{F}$, which drives the flow at constant bulk velocity, are non-dimensionalized with the bulk velocity $U_{b}$ and the pipe diameter $D$ as follows:

$$
U_{b}=\frac{1}{A} \int_{A} U_{z} \mathrm{dA}, \quad \tilde{\mathbf{U}}=\frac{\mathbf{U}}{U_{b}}, \quad \tilde{t}=\frac{t U_{b}}{D}, \quad \tilde{\mathbf{x}}=\frac{\mathbf{x}}{D}, \quad \tilde{p}=\frac{p}{\rho U_{b}^{2}}, \quad \tilde{\mathbf{F}}=\frac{\mathbf{F} D}{U_{b}^{2}}
$$

The cross section of the pipe is given by $A$ and $U_{z}$ is the velocity component in streamwise direction. The bulk Reynolds number and the Péclet number are defined as $R_{b}=U_{b} D / \nu$ and $P e=U_{b} D / \alpha$, respectively. The nondimensional temperature is defined as a scaled temperature difference, employing time-averaged quantities denoted by $\langle\cdot\rangle^{t}$,

$$
\Theta=\frac{\left\langle T_{w}\right\rangle^{t}-T}{\left\langle q_{w}\right\rangle^{t}} \rho c_{p} U_{b}=\frac{\left\langle T_{w}\right\rangle^{t}-T}{\left\langle T_{w}\right\rangle^{t}-T_{b}} \frac{P e}{N u}, \quad T_{b}=\frac{\int_{A}\left\langle U_{z} T\right\rangle^{t} \mathrm{~d} \mathrm{~A}}{\int_{A}\left\langle U_{z}\right\rangle^{t} \mathrm{dA}}
$$

because in the thermally fully developed region the condition of $\partial\langle\Theta\rangle^{t} / \partial z=0$ 155 (as well as $N u=$ const.) is satisfied [7, 18. Therefore, periodic boundary conditions are also admissible for the thermal field. Wall temperature, wall heat flux and bulk temperature [19] are denoted by $T_{w}, q_{w}$ and $T_{b}$, respectively. 
The Nusselt number is defined in the usual way as

$$
N u=\frac{q_{w} D}{\left(\left\langle T_{w}\right\rangle^{t}-T_{b}\right) \lambda}
$$

The three types of thermal boundary conditions (MBC, IF, IT) differ both in the quantity being imposed at the wall

$$
\begin{array}{rlrl}
T_{w} & =\text { const. } & \Theta=0 & \text { MBC, IT } \\
q_{w}=\lambda \frac{\partial T}{\partial r}=\text { const. } & \frac{\partial \Theta}{\partial \tilde{r}}=-P e & \text { IF }
\end{array}
$$

as well as in the source term $S_{\Theta}$

$$
S_{\Theta}= \begin{cases}4 \tilde{U}_{z} & \text { MBC, IF } \\ \tilde{a} \Theta \tilde{U}_{z}-\frac{1}{P e}\left(2 \tilde{a} \frac{\partial \Theta}{\partial \tilde{z}}-\tilde{a}^{2} \Theta\right) & \text { IT }\end{cases}
$$

stemming from the non-dimensionalization [7. For MBC and IF, the temperature varies linearly in streamwise direction [19. The implementation of the IT boundary condition follows Piller [7. For IT, an exponential decay in streamwise direction of the time-averaged difference between bulk and wall temperature is found for negligible net axial conduction [7, 19].

$$
\frac{\left\langle T_{w}\right\rangle^{t}-T_{b}}{\left(\left\langle T_{w}\right\rangle^{t}-T_{b}\right)_{\tilde{z}=0}}=\exp (-\tilde{a} \tilde{z})
$$

According to Weigand [20, the effect of axial heat conduction can be neglected for $P e>100$. For the present simulation of $\operatorname{Pr}=0.025$ the Péclet numbers range from $P e=132.5$ to $P e=942.5$. The exponential decay rate is given by a. A priori, this term is unknown. Therefore, it is evaluated at runtime such that the integral energy balance is satisfied, as proposed by Piller 7 . For the LES of $R e_{b}=5300$ the mean and standard deviation for the exponential decay rate are $(0.018732,8.682219 e-05)$ for $\operatorname{Pr}=0.71$ and $(0.156291,8.103444 e-$ 04) for $\operatorname{Pr}=0.0 .25$. For $\operatorname{Re}_{b}=37700$ the mean and standard deviation are $(0.012472,6.440540 e-05)$ for $\operatorname{Pr}=0.71$ and $(0.039238,1.909494 e-04)$ for $\operatorname{Pr}=0.025$. Therefore, due to the averaging in time, necessary anyway for 
converged statistics, the uncertainty in the exponential decay rate is sufficiently small.

Instantaneous values of velocity and temperature are decomposed into mean and fluctuating parts according to

$$
U=\langle U\rangle+u, \quad \Theta=\langle\Theta\rangle+\vartheta
$$

If no explicit superscript is given for the averaging, it is performed in time and over the homogeneous directions $z$ and $\varphi$. Note that the turbulence statistics in this work were computed using the Nek5000 toolbox developed by Vinuesa et al. 21. As discussed in Ref. 21, the statistics are collected at runtime, and they are averaged over time and streamwise direction. In a postprocessing step, the averaging in azimuthal direction is performed. Turbulent velocity fields are generated based on a laminar solution with superimposed perturbations [22] using a polynomial order of $N=5$. The generated field is fed into a simulation with $N=7$ as initial condition. After a fully-developed state is reached, we begin collecting statistics. The required averaging periods are found in a prestudy where convergence indicators like the deviation of the total shear stress from the analytical one 23] and the residual of the total heat flux balance [24] are evaluated together with visual inspection of the convergence of the statistics.

The bulk Reynolds number is varied within the range $R e_{b}=\{5300,11700$, 19000, 37700\}, corresponding to friction Reynolds numbers of $R e_{\tau} \approx\{180$, $360,550,1000\}$. Prandtl number values of $\operatorname{Pr}=0.71$, representing air, and $\operatorname{Pr}=0.025$, representing a class of liquid metals such as lead-bismuth eutectic, mercury or gallium-indium-tin [25, are selected. In case of $R e_{b}=5300$, a DNS has been conducted with a resolution in streamwise, azimuthal and wall-normal direction of $\Delta z^{+}<9.4, \Delta(R \varphi)^{+}<4.9$ and $\Delta y^{+}<4.5$, respectively, similar to the one employed by El Khoury et al. [26] to setup a reference case. The superscript ${ }^{+}$denotes scaling in viscous units, i.e. with the friction velocity $u_{\tau}$ and kinematic viscosity $\nu$. The first point away from the wall is at $\Delta y_{1}^{+}<$ 0.37. Well-resolved large-eddy simulations (LESs) are performed for the bulk 


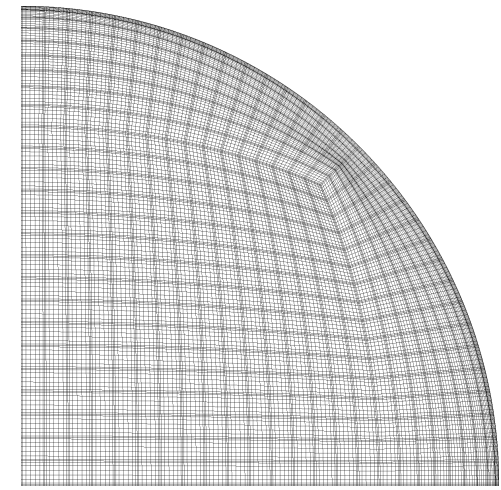

Figure 1: Quarter of the cross section of the employed spectral element mesh for the LES at $R e_{b}=37700$.

Reynolds numbers of $R e_{b}=\{5300,11700,19000,37700\}$. Due to the larger thermal scales for $\operatorname{Pr}=0.025$, the thermal field is still properly resolved for such low Prandtl numbers [27, 28]. The additional dissipation, which needs to be added to the governing equations, is based on a variant of the approximate deconvolution model presented by Schlatter et al. 29 and recently tested and applied in an LES of an airfoil by Negi et al. 30. An additional relaxation term, responsible for the required dissipation, given by $-\chi \mathscr{H}(u)$ and $-\chi \mathscr{H}(\Theta)$ is added to the right hand side of the momentum and temperature Eqs. (1) and (2), respectively. Here, $\mathscr{H}$ denotes a high-pass spectral filter (applied to the respective field) and $\chi$ is a model parameter, adjusted for each Reynolds number according to previous studies [30]. The resolution criteria proposed by Negi et al. 30] are adopted (with a slightly coarser resolution in azimuthal direction) so that $\Delta z^{+}<18, \Delta(R \varphi)^{+}<10, \Delta y^{+}<11$, and $\Delta y_{1}^{+}<0.64$ and validated by comparing velocity and thermal statistics at $R e_{b}=5300$ with the results of the present DNS as well as a comparison with literature data at $R e_{b}=5300$ and $R e_{b}=37700$ presented in the following subsection.

Fig. 1 shows the distribution of the elements within a quarter of the cross section for the LES at $R e_{b}=37700$. Note that the full cross section is used for the simulations. The total number of elements and grid-points for each 

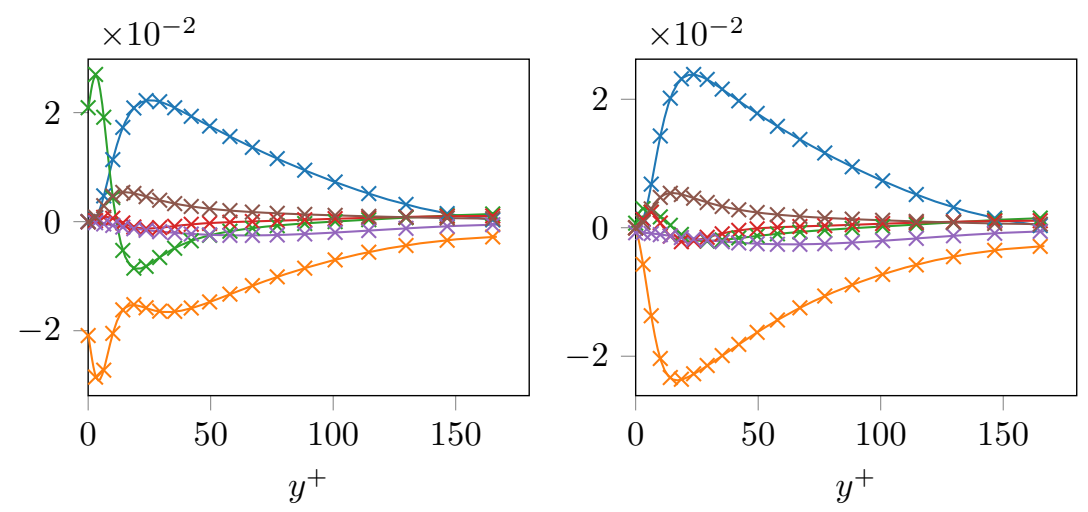

Figure 2: Budget of the turbulent heat flux $\left\langle u_{z} \vartheta\right\rangle$ at $R e_{b}=5300$ for reference DNS: - and LES: $\quad \times$ at $P r=0.025$ of (left) MBC and (right) IF. Individual contributions are $P^{+}:-$ $, \epsilon^{+}:-, M D^{+}:-, T D^{+}:-, T P G^{+}:-, S^{+}:-$as defined in Appendix $\mathrm{A}$

simulation is summarized in Table 1 together with the time-step size $\Delta t$. The

Table 1: Number of elements per cross section $n e l_{C S}$, streamwise direction $n e l_{z}$ and total number of grid points together with the time-step size $\Delta t$ and the averaging time $t_{a}$.

\begin{tabular}{lrrrrr}
\hline$R e_{b}$ & nel $_{C S}$ & nel $_{z}$ & grid-points & $\Delta t U_{b} / D$ & $t_{a} U_{b} / D$ \\
\hline $5300(\mathrm{LES})$ & 132 & 54 & 3649536 & $2 \times 10^{-3}$ & 8000 \\
$5300(\mathrm{DNS})$ & 384 & 100 & 19660800 & $1 \times 10^{-3}$ & 3600 \\
$11700(\mathrm{LES})$ & 432 & 105 & 23224320 & $1 \times 10^{-3}$ & 1600 \\
$19000(\mathrm{LES})$ & 828 & 160 & 67829760 & $5 \times 10^{-4}$ & 1675 \\
$37700(\mathrm{LES})$ & 2176 & 290 & 323092480 & $4 \times 10^{-4}$ & 212 \\
\hline
\end{tabular}

time-step is kept constant in each simulation at a value so that $C F L \lesssim 0.5$.

\subsection{Validation \& Domain Size}

The agreement between statistics of the reference DNS and the well-resolved LES at $R e_{b}=5300$ is exemplified in Fig. 2. The individual contributions to the budget of the streamwise turbulent heat flux between reference DNS 


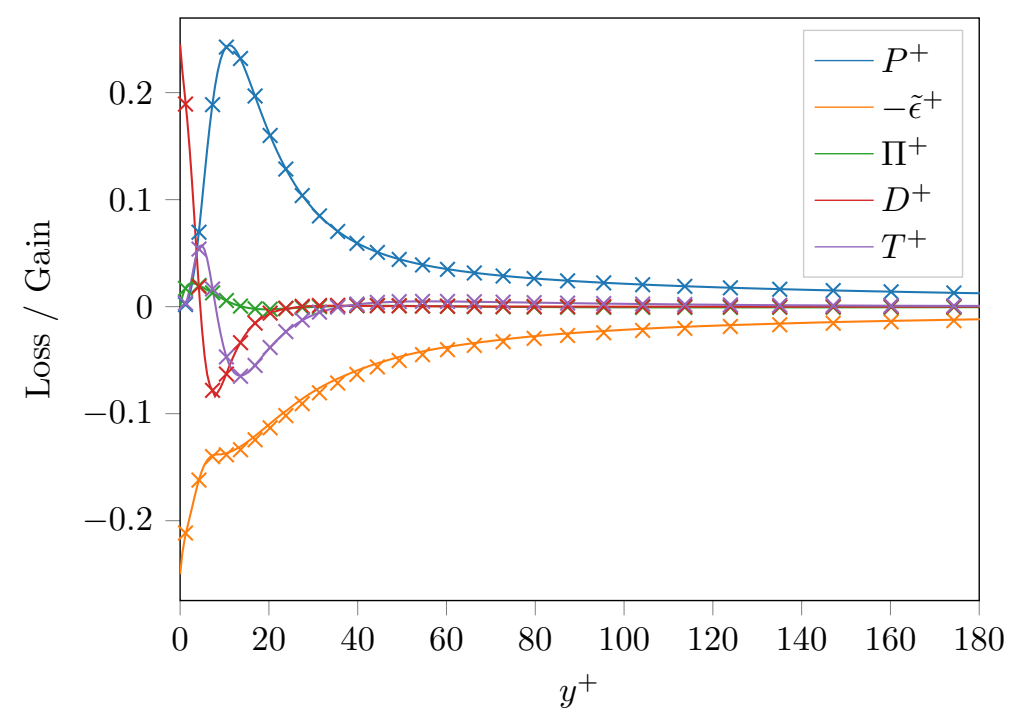

Figure 3: Budget of turbulent kinetic energy at $R e_{b}=37700$ compared to reference DNS data by El Khoury et al. [26]: $\quad \times$ scaled in viscous units. Individual contributions are defined in Appendix A

and LES are indistinguishable. Similarly good agreement is found for other statistics (not shown). Therefore, for $R e_{b}>5300$ only LESs are performed. It is interesting to note the different behavior of dissipation $\epsilon$ and molecular diffusion MD contributions close to the wall due to the imposition of a nonfluctuating temperature boundary condition in MBC, as is found by Flageul et al. [8] in channel flow.

For higher Reynolds numbers, velocity statistics of the present LES are compared to literature data from El Khoury et al. [26]. The friction Reynolds number of the present LES, i.e. $R e_{\tau}=998.9$, agrees well with that of the DNS [26], which is $R e_{\tau}=999.0$. The budget of the turbulent kinetic energy for the case at the highest considered Reynolds number of $R e_{b}=37700$ is shown in Fig. 3 The individual contributions are defined as in El Khoury et al. [26] and restated for completeness in Appendix A. Note that the wall-normal location is limited 245 to $0<y^{+}<180$, since for $y^{+}>180$ mainly production and dissipation balance [26]. Except for a slightly too low dissipation of the present LES (maximum 7\% 

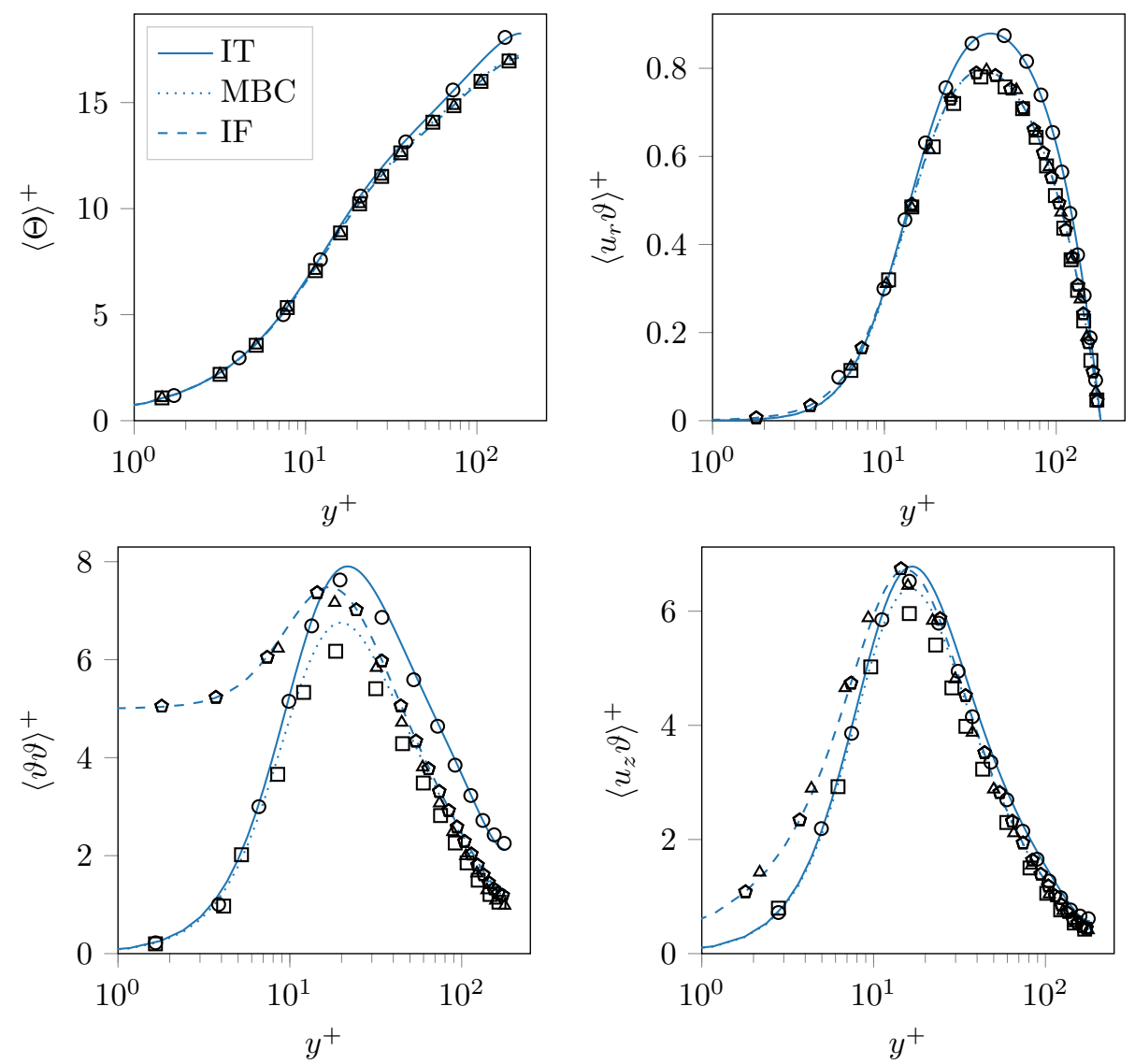

Figure 4: Mean temperature, radial turbulent heat flux, temperature variance and streamwise turbulent heat flux scaled in viscous units at $\operatorname{Re}_{b}=5300$ and $\operatorname{Pr}=0.71$ compared to Piller [7] (IT: O, MBC: $\square$, IF: $\Delta$ ) and Antoranz et al. 31] (IF: ๑)

lower in magnitude), the agreement is excellent. Based on this agreement we term the present LES well-resolved.

In order to validate the implementation of the thermal boundary conditions, the present results are compared to literature data. Fig. 4 shows the mean temperature $\langle\Theta\rangle^{+}$, the temperature variance $\langle\vartheta \vartheta\rangle^{+}$and the turbulent heat fluxes in radial and streamwise direction $\left\langle u_{r} \vartheta\right\rangle^{+},\left\langle u_{z} \vartheta\right\rangle^{+}$, respectively. The mean temperature and radial turbulent heat flux show good agreement with data from Piller [7] for all TBCs considered. Peak values of temperature variance and 
streamwise turbulent heat flux appear to be underpredicted by Piller [7] who applied a second order finite volume method in a rather short pipe of $6.328 \mathrm{D}$. A more recent simulation for IF by Antoranz et al. [31, who applied the same high-order method as the one considered in this work for the same domain size, shows good agreement with the present results also for the peak values of temperature variance and streamwise turbulent heat flux.

Another issue is to select the domain size appropriately such that the periodic boundary conditions do not affect the thermal and velocity statistics artificially. In general, the domain size should be large enough to accommodate the largest thermal and flow structures. Two studies dedicated to pipe length requirements are by Chin et al. 32] and Saha et al. 33]. The second study concluded that for thermal statistics, using MBC at a friction Reynolds number of $R e_{\tau} \approx 170$ and Prandtl numbers of $\operatorname{Pr}=0.025,0.71,2.0$, a pipe length of $L_{z}=8 \pi R \approx 12.5 D$ is sufficient to accurately capture up to fourth-order statistics as well as twopoint correlations. In a recent study considering the computational domain for MBC in a channel flow for $R e_{\tau}=500,1000$ and $\operatorname{Pr}=0.71$, Lluesma-Rodríguez et al. 24] concluded that even a smaller domain of $2 \pi h, 2 h, 2 \pi h$ in streamwise, wall-normal and spanwise direction may be enough to obtain accurate one-point statistics and turbulent budgets, even though the domain is too small for the largest thermal structures. As the previously mentioned studies do not consider 275 IT and IF types of TBC, the streamwise two-point correlations for the thermal fields are assessed in the present work to check for an appropriate domain length. They are shown for $R e_{b}=5300$ and $R e_{b}=11700$ in Fig. 5. Evidently, the two-point correlations for all cases, except for the case of IT at $R e_{b}=5300$ and $\operatorname{Pr}=0.025$, vanish at a separation of half the pipe length $\Delta z=6.25 D$, indicating a sufficiently long domain length of $L_{z}=12.5 \mathrm{D}$. Additionally, an LES of a four times longer domain of $L_{z}=50 D$ shows that even though the streamwise two-point correlations for IT at $P r=0.025$ do not vanish, the Nusselt number, mean temperature and turbulent heat fluxes are unaffected. Only the temperature variance at the lower Prandtl number is altered by the longer domain, resulting in a $5 \%$ increase of the fluctuations occurring at the 

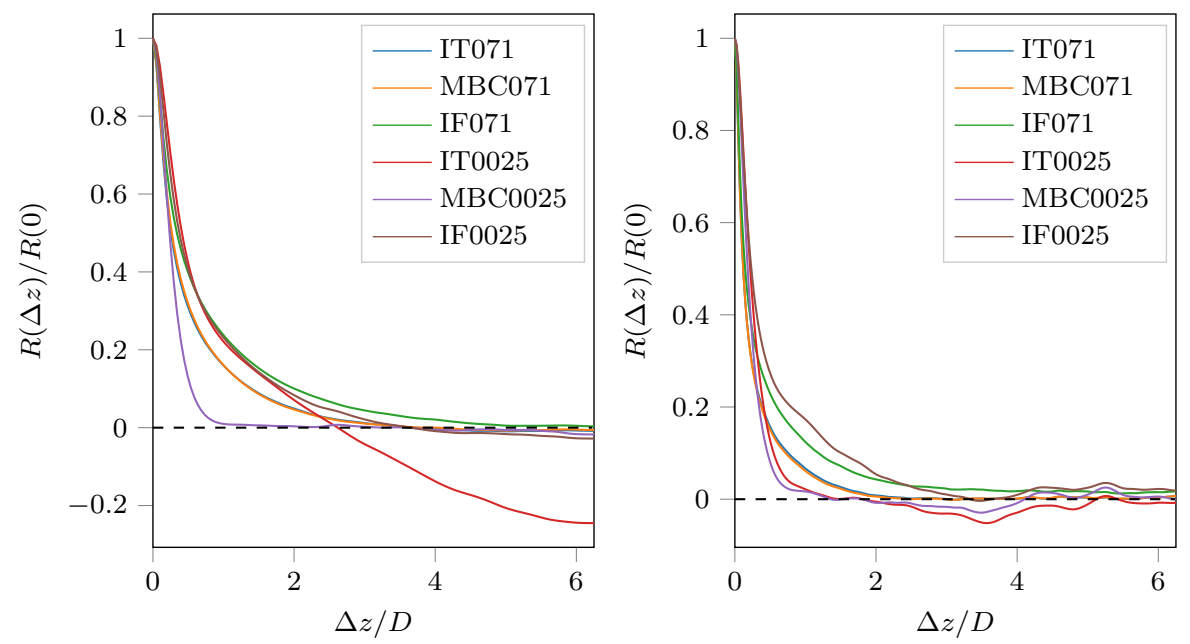

Figure 5: Streamwise two-point correlations of thermal fields at wall-normal location of maximum temperature variance for (left) $R e_{b}=5300$ and (right) $R e_{b}=11700$.

wall for IF, as found by Tiselj [34]. For the IT boundary condition at $\operatorname{Pr}=$ 0.025 the two-point correlations of the temperature fluctuations do not vanish in the longer domain either and the temperature variance in the center of the pipe is about twice as large as in the domain length of $L_{z}=12.5 \mathrm{D}$. Thus, the temperature variance and power spectral density of the isothermal case at $R e_{b}=5300$ and $\operatorname{Pr}=0.025$ are excluded from the evaluation in the following section.

An explanation for the non-vanishing two-point correlations of IT, occurring only at the lowest considered Reynolds and Prandtl numbers, most probably is that the assumption of negligible net axial heat conduction, yielding the exponential decay of the temperature difference in IT, Eq. (9), is not valid for such low Péclet numbers and hence the derived source term in Eq. 8) is not correct for this particular case. For higher Reynolds numbers, as exemplified on the right diagram for $R e_{b}=11700$, all correlations drop to zero within half of the domain length. 


\section{Results and Discussion}

The following subsections summarize the main findings in terms of (decomposed) Nusselt number, first- and second-order statistics and spectral analysis.

\subsection{Nusselt Number}

305 predict the Nusselt number such as the Gnielinski correlation for fully-developed turbulent flow valid in the range of $10^{4}<\operatorname{Re}_{b}<10^{6}$ and $0.1<\operatorname{Pr}<1000$ 35. The range of validity can be extended up to lower Reynolds numbers $\left(R e_{b}=\right.$ 2300) by interpolation of laminar and turbulent Nusselt numbers as described

numbers. Two recommended correlations [36] for IF are the ones by Skupinski et al. 38 (measured Range of $P e=58-13100$ for NaK in hydrodynamically and thermally fully-developed horizontal pipe flow) and Lubarsky and Kaufman [39] (best fit of experimental data). Prandtl number, the different types of TBCs do not influence the Nusselt number and they are accurately predicted by the Gnielinski correlation. This can be explained by the dominance of turbulent mixing over conduction for 

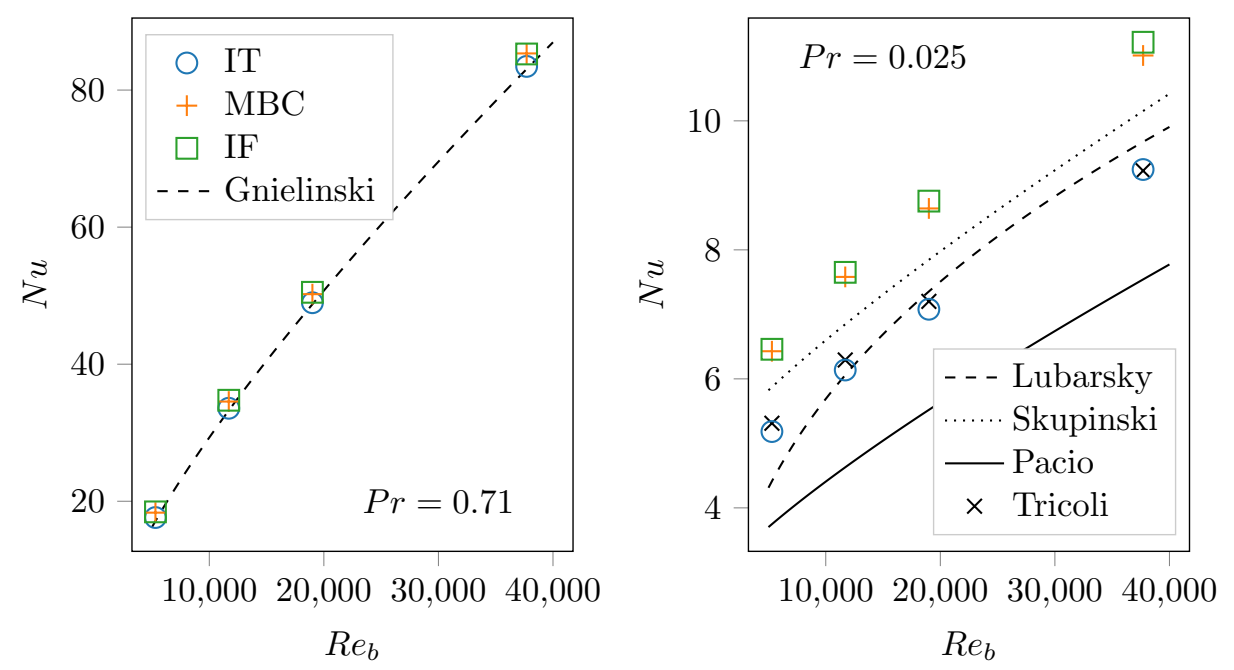

Figure 6: Nusselt number variation with $R e_{b}$.

330

medium and high Prandtl numbers (as is discussed in the following section for $\mathrm{MBC}$ and IF). The right figure shows the Nusselt number for $\operatorname{Pr}=0.025$. For this Prandtl number, the present results show a significant effect of the TBC even on a global quantity as the Nusselt number. The Nusselt numbers of MBC agree well with IF, but the Nusselt number of IT is significantly

lower. For such low Prandtl numbers and moderately high Reynolds numbers, typical of engineering applications, the contribution of conduction to the heat transfer is comparable to that of turbulent mixing, resulting in different Nusselt numbers depending on the applied boundary condition at the wall. This is analogous to the laminar convective case, where for fully-developed lami340 Reynolds numbers of $R e_{b}=\{5300,11700,19000,37700\}$ the Nusselt number of IT is $\{19.8 \%, 19.7 \%, 19.2 \%, 17.5 \%\}$ lower than that for IF and MBC, respectively, indicating a converging trend for higher Reynolds numbers. A possible explanation for this trend is the dominance of turbulent mixing over molecu345 lar conduction for increasing Reynolds numbers, as observed for $\operatorname{Pr}=0.71$. 
For a conclusive statement however, even higher Reynolds numbers should be investigated for low-Prandtl-number fluids.

Besides, the correlations by Lubarsky and Kaufman [39] and Skupinski et al. 38. for IF underpredict the Nusselt numbers as well as the one for IT from Pacio 350 et al. [36. The theoretical relationship between the Nusselt numbers of IF and IT from Tricoli [37] however, predicts the Nusselt numbers of IF well (based on the present values of IT). According to the present Nusselt number results, more accurate correlations for liquid metals, eventually based on reliable experimental or numerical data, are necessary. Although the numerical results in this study may not be sufficient to propose a new correlation, they serve as a reference to test state-of-the-art correlations and extend the openly available data for liquid metals.

\subsection{Decomposition of the Nusselt Number}

Similarly to the Fukagata-Iwamoto-Kasagi (FIK) identity for the skin-friction coefficient [40, also the Nusselt number can be decomposed into laminar and turbulent contributions [41, 42]. A derivation of the decomposition for the $\mathrm{MBC} / \mathrm{IF}$ boundary condition in forced convection, fully-developed turbulent pipe flow is shown in Appendix B. Using the non-dimensionalization listed in Section 2, the Nusselt number is decomposed into

$$
\begin{aligned}
\frac{1}{N u} & =\underbrace{\frac{11}{48}}_{1 / N u_{L}}-\underbrace{\int_{0}^{0.5}(1+\tilde{\Phi})\left\langle\tilde{u}_{r} \vartheta\right\rangle \mathrm{d} \tilde{r}}_{1 / N u_{H F, r}} \\
& -\underbrace{\frac{1}{2} \int_{0}^{0.5} \frac{1}{\tilde{r}}\left[\left(2 \tilde{r}^{4}-\tilde{r}^{2}\right) 16 \tilde{\Phi}_{T}-\tilde{\Phi}_{T}^{2}\right] \mathrm{d} \tilde{r}}_{1 / N u_{R S S}}+\underbrace{\frac{8}{P e} \int_{0}^{0.5}\left\langle\tilde{u}_{z} \vartheta\right\rangle \tilde{r} \mathrm{~d} \tilde{r}}_{1 / N u_{H F, z}}
\end{aligned}
$$

The four contributions to the inverse Nusselt number in eq. (11) are the laminar term $N u_{L}$, the contribution due to the radial turbulent heat flux $N u_{H F, r}$, the contribution due to the turbulent velocity field $N u_{R S S}$ (where $\tilde{\Phi}$ is the fractional flow rate of the velocity field and $\tilde{\Phi}_{T}$ the fractional flow rate of the turbulent velocity field, as described in Appendix $\mathrm{B}$ and the contribution due to the 


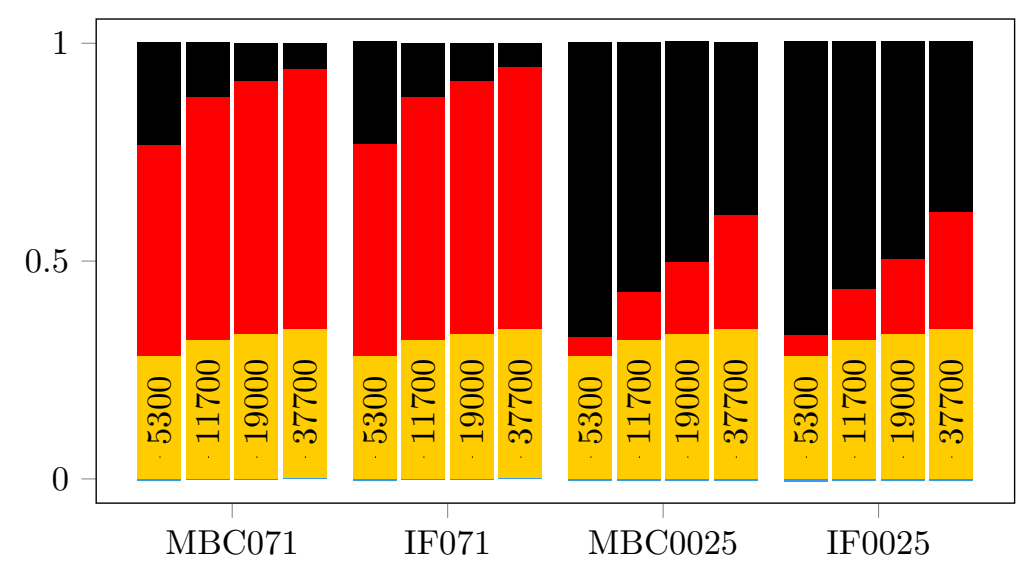

Figure 7: Individual contributions to the Nusselt number: $N u_{L} / N u$ : $\boldsymbol{\square}, N u_{L} / N u_{H F, r}$ : , $N u_{L} / N u_{R S S}: \quad, N u_{L} / N u_{H F, z}$ :

streamwise turbulent heat flux $N u_{H F, z}$, respectively. Since the turbulent velocity field can be expressed by the Reynolds shear stress (RSS) (see Appendix B), the fractional flow rate $\Phi_{T}$ and consequently $N u_{R S S}$ are only affected by the RSS and no thermal statistics. Note that due to the inverse relationship, the individual contributions cannot be summed together to determine the Nusselt number in contrast to the contributions of the decomposed skin friction coefficient [40. For instance, a higher radial turbulent heat flux increases the second term on the right hand side of eq. (11), so that a higher value is subtracted from $11 / 48$, thereby reducing the right hand side of eq. (11). This results in an increase of the Nusselt number because of the inverse relationship. In order to visualize and compare the individual contributions and to highlight the different heat transfer mechanism of low-Prandtl-number fluids, eq. (11) is reformulated as

$$
1=\frac{N u_{L}}{N u}+\frac{N u_{L}}{N u_{H F, r}}+\frac{N u_{L}}{N u_{R S S}}-\frac{N u_{L}}{N u_{H F, z}}
$$

with the individual ratios shown in in Fig. 7. Note that a higher Nusselt number (i.e. lower $N u_{L} / N u$ ) is achieved by larger ratios $N u_{L} / N u_{H F, r}$ and $N u_{L} / N u_{R S S}$. The separate analysis of each of the three turbulent contributions highlights the different heat transfer mechanism of the two investigated Prandtl 
number fluids. Note that the contribution due to the streamwise turbulent heat flux is one to two orders of magnitude smaller than the other contributions and its effect on the Nusselt number is negligible for the present simulations. The third term in eq. (12) only depends on the velocity field through the Reynolds shear stresses and is thus independent of the Prandtl number for forced convection. For the Reynolds numbers considered in the present study, it ranges from $28 \%$ to $34 \%$. The ratio determining the contribution due to the radial turbulent heat flux $N u_{L} / N u_{H F, r}$ is the dominant contribution to the Nusselt number for $\operatorname{Pr}=0.71$ with values ranging from $48 \%$ to $60 \%$. For $\operatorname{Pr}=0.025$, the contribution due to the radial turbulent heat flux only becomes relevant for higher Reynolds numbers with values of $N u_{L} / N u_{H F, r}$ ranging from $4 \%$ to $27 \%$. Therefore, for the Reynolds numbers considered in the present study, the increase in Nusselt number for $\operatorname{Pr}=0.025$ with respect to the laminar Nusselt number is mainly caused by the contribution due to the turbulent velocity field and not the contribution due to the radial turbulent heat flux.

The individual contributions, the Nusselt number defined in eq. (11) and the Nusselt number evaluated directly by the bulk temperature as shown in Appendix $\mathrm{B}$ are given in Table 2. The agreement between the directly evaluated Nusselt number and the one from eq. (11) is good in all cases except for $\operatorname{Pr}=0.71$ at $R e_{b}=37700$ where the Nusselt number is underestimated. This discrepancy could be caused by the high sensitivity of the Nusselt number evaluated using Eq. (11) on the radial turbulent heat flux distribution for high Reynolds numbers. Due to the inverse relation given by Eq. [11, the determined Nusselt number becomes more sensitive to deviations in $1 / N u_{H F, r}$ as the sum of the three turbulent contributions approaches its upper bound 11/48. For the cases of $R e_{b}=37700$ and $\operatorname{Pr}=0.71$ an amplified $\left\langle\tilde{u}_{r} \vartheta\right\rangle$ of $\approx 1 \%$ yields the correct Nusselt number illustrating its high sensitivity. Additionally, the influence of the contribution due to the turbulent velocity field is tested. When using the DNS data of El Khoury et al. 26] for these two cases to determine $1 / N u_{R S S}$ and the fractional flow rate $\tilde{\Phi}$, the Nusselt number is still underestimated, so that indeed a slightly too low radial turbulent heat flux could be 
Table 2: Individual contributions, Nusselt number defined in eq. 11 and Nusselt number evaluated directly in parentheses. The bulk Reynolds number increases from top to bottom $\left(R e_{b}=5300,11700,19000,37700\right)$.

\begin{tabular}{|c|c|c|c|c|}
\hline & $1 / N u_{H F, r}$ & $1 / N u_{R S S}$ & $1 / N u_{H F, z}$ & $N u$ \\
\hline MBC071 & $1.11 \times 10^{-1}$ & $6.45 \times 10^{-2}$ & $7.68 \times 10^{-4}$ & $18.3(18.3)$ \\
\hline IF071 & $1.11 \times 10^{-1}$ & $6.45 \times 10^{-2}$ & $8.13 \times 10^{-4}$ & $18.4(18.5)$ \\
\hline MBC0025 & $9.86 \times 10^{-3}$ & $6.45 \times 10^{-2}$ & $8.04 \times 10^{-4}$ & $6.43(6.43)$ \\
\hline IF0025 & $1.09 \times 10^{-2}$ & $6.45 \times 10^{-2}$ & $1.16 \times 10^{-3}$ & $6.46(6.46)$ \\
\hline MBC071 & $1.27 \times 10^{-1}$ & $7.31 \times 10^{-2}$ & $2.67 \times 10^{-4}$ & $34.5(34.5)$ \\
\hline IF071 & $1.28 \times 10^{-1}$ & $7.31 \times 10^{-2}$ & $2.78 \times 10^{-4}$ & $34.7(34.7)$ \\
\hline MBC0025 & $2.49 \times 10^{-2}$ & $7.31 \times 10^{-2}$ & $8.05 \times 10^{-4}$ & $7.58(7.58)$ \\
\hline IF0025 & $2.64 \times 10^{-2}$ & $7.31 \times 10^{-2}$ & $1.04 \times 10^{-3}$ & $7.65(7.65)$ \\
\hline MBC071 & $1.33 \times 10^{-1}$ & $7.59 \times 10^{-2}$ & $1.50 \times 10^{-4}$ & $50.2(50.2)$ \\
\hline IF071 & $1.34 \times 10^{-1}$ & $7.59 \times 10^{-2}$ & $1.56 \times 10^{-4}$ & $50.4(50.5)$ \\
\hline MBC0025 & $3.83 \times 10^{-2}$ & $7.59 \times 10^{-2}$ & $7.91 \times 10^{-4}$ & $8.64(8.64)$ \\
\hline IF0025 & $4.00 \times 10^{-2}$ & $7.59 \times 10^{-2}$ & $1.00 \times 10^{-3}$ & $8.75(8.75)$ \\
\hline MBC071 & $1.37 \times 10^{-1}$ & $7.85 \times 10^{-2}$ & $7.15 \times 10^{-5}$ & $74.5(85.3)$ \\
\hline IF071 & $1.38 \times 10^{-1}$ & $7.85 \times 10^{-2}$ & $7.47 \times 10^{-5}$ & $79.0(85.3)$ \\
\hline MBC0025 & $6.00 \times 10^{-2}$ & $7.85 \times 10^{-2}$ & $7.12 \times 10^{-4}$ & $11.0(11.0)$ \\
\hline IF0025 & $6.12 \times 10^{-2}$ & $7.85 \times 10^{-2}$ & $8.72 \times 10^{-4}$ & $11.1(11.2)$ \\
\hline
\end{tabular}

responsible for the observed discrepancy.

A decomposition for IT was not found due to the complex source term in Eq. (8), but the mean temperature budget, discussed in the following subsection, includes IT. Nevertheless, an explanation for the significant differences between the Nusselt numbers of IT and MBC/IF can be inferred because also for IT, it can be assumed that the Nusselt number consists of a laminar and a turbulent contribution. The laminar contribution, which prevails in determining the Nusselt number for low Prandtl numbers at the Reynolds numbers considered here, 


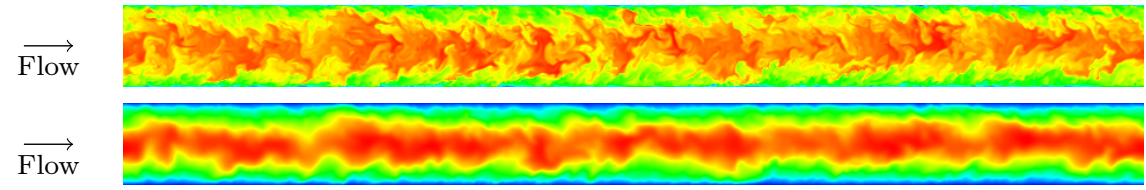

Figure 8: Instantaneous visualisations of the temperature $\Theta$ at $R e_{b}=19000$ of MBC at (top) $\operatorname{Pr}=0.71$ and at (bottom) $P r=0.025$, scaled with individual maximum. Red (blue) regions indicate high (low) values.

Table 3: Line styles and colors of Reynolds numbers and thermal boundary conditions.

\begin{tabular}{ccc} 
& $R e_{b}=5300$ & $R e_{b}=37700$ \\
\hline IT & - & - \\
MBC & $\cdots \ldots$ & $\cdots$ \\
IF & --- & --- \\
\hline
\end{tabular}

is lower for IT than for MBC/IF, such that also the Nusselt number is lower.

Even though the Nusselt numbers for $\operatorname{Pr}=0.025$ are larger than analytically found for the laminar case, the thermal field is still in a laminar-like state. Such a laminar-like thermal field can be observed in the instantaneous snapshots in ${ }_{430}$ Fig. 8 which correspond to the $\mathrm{MBC}$ at $\operatorname{Pr}=0.71$ and at $\operatorname{Pr}=0.025$ at $R e_{b}=19000$. Indeed, while for $P r=0.71$ large and small scales with sharp gradients are recognizable, for $\operatorname{Pr}=0.025$ the small scales are smeared out by the high thermal diffusivity [34, resulting in a much smoother temperature field, resembling a laminar one.

\subsection{Turbulence Statistics}

In addition to the effect of the TBCs on the Nusselt number as a global parameter, first- and second-order one-point statistics are compared in the following paragraphs. To distinguish the TBCs in the following diagrams, different line styles are used while the Reynolds number is distinguished by the line color, as summarized in Table 3 . In order to keep the different profiles for each TBC and Reynolds number clearly distinguishable, the following diagrams only show 

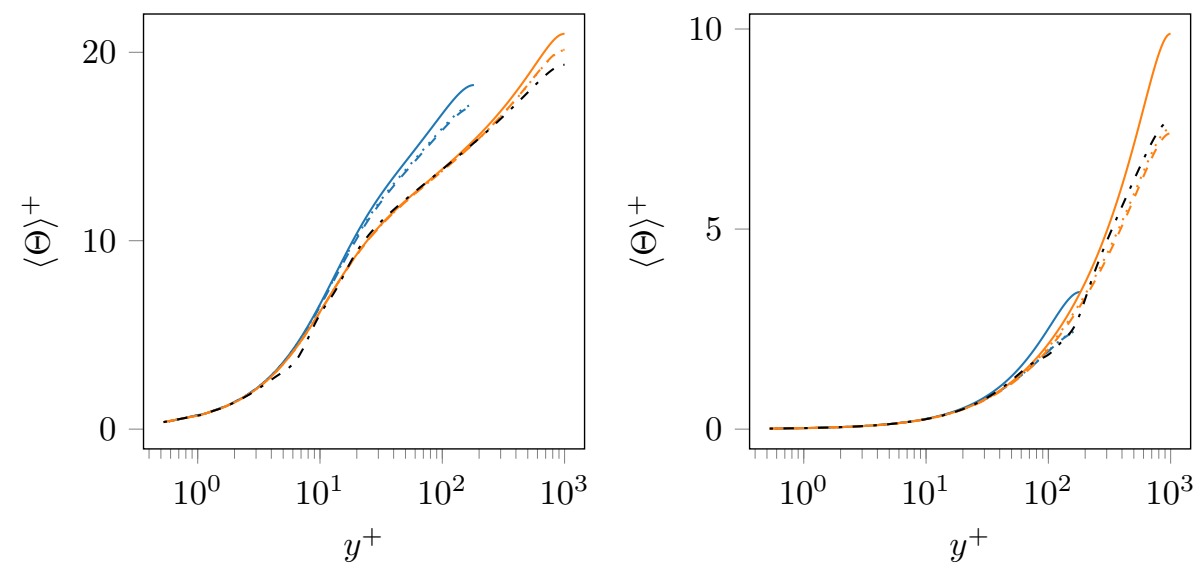

Figure 9: Mean temperature variation with $\mathrm{TBC}$ and $R e_{b}$ for (left) $\operatorname{Pr}=0.71$ and (right) $\operatorname{Pr}=0.025$. Line styles and colors as in Table 3 Empirical correlation by Kader [44]: - - .

results for lowest and highest Reynolds number. Results of the intermediate Reynolds numbers are provided in the database [43].

The temperature distribution is presented in Fig. 9. Scaling in viscous units is done by substituting the bulk velocity $U_{b}$ by the friction velocity $u_{\tau}$ in the definition of the non-dimensionalized temperature in eq. (4), i.e. $\Theta^{+}=\Theta u_{\tau} / U_{b}$. For both Prandtl numbers, MBC and IF yield very similar temperature profiles irrespective of the Reynolds number. However, the temperature profile of IT deviates from the other two by higher values in the core region (cf.[7]), an effect which is especially pronounced at $\operatorname{Pr}=0.025$. A higher temperature for IT compared to $\mathrm{MBC} / \mathrm{IF}$ in the center center of the pipe at low Prandtl numbers $\left(\approx 33 \%\right.$ at $\left.R e_{b}=37700\right)$ reflects the previously discussed lower Nusselt numbers of IT. Neither buffer layer nor logarithmic layer are visible for $\operatorname{Pr}=0.025$ (cf. [4]). The empirical correlation by Kader [4] (only shown for $R e_{b}=37700$ ) agrees well with the present data for IF/MBC at both Prandtl numbers (cf. [4]). 

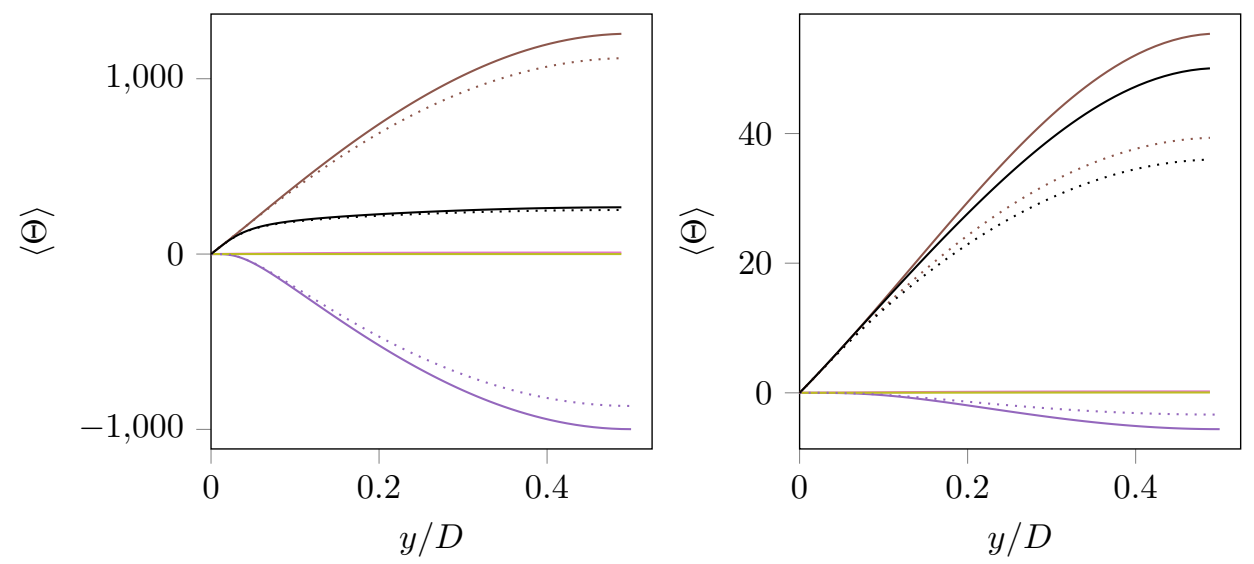

Figure 10: Mean temperature budget of IT and MBC at $R e_{b}=11700$ (left) $\operatorname{Pr}=0.71$ and (right) $\operatorname{Pr}=0.025$. Contributions are due to radial turbulent heat flux: - , mean advective heat flux: - $\quad$ turbulent axial advective heat flux: — and mean streamwise thermal diffusion: - Their sum is equal to $\langle\Theta\rangle$ : — . Line styles as in Table 3

Even though a decomposition of the Nusselt number for IT was not found, the budget equation for the mean temperature given by Ref. [7] reads:

$$
\langle\Theta\rangle^{t}(\tilde{r})=-P e \int_{r^{\prime}=\tilde{r}}^{0.5}\left\langle\tilde{u}_{r} \vartheta\right\rangle \mathrm{dr}^{\prime}+P e \int_{r^{\prime}=\tilde{r}}^{0.5} \frac{1}{r^{\prime}} \int_{r^{\prime \prime}=0}^{r^{\prime}} r^{\prime \prime}\left\langle S_{\Theta}\right\rangle \mathrm{dr}^{\prime \prime} \mathrm{dr}^{\prime}
$$

Eq. 13. can be used to analyze the different contributions to the mean temperature, as illustrated in Fig. 10 for $R e_{b}=11700$ and both IT and MBC, while IF is not shown because it practically coincides with MBC. The dominant terms are the contributions due to the radial turbulent heat flux $\left\langle\tilde{u}_{r} \vartheta\right\rangle$ and the mean advective heat flux stemming from the source term, $4\left\langle\tilde{U}_{z}\right\rangle$ for MBC and IF, and $\tilde{a}\left\langle\tilde{U}_{z}\right\rangle\langle\Theta\rangle$ for IT. Their difference determines the distribution of $\langle\Theta\rangle$ and hence its dependency on the TBC. For both Prandtl numbers, the contributions due to turbulent axial advective heat flux $\tilde{a}\left\langle\tilde{u}_{z} \vartheta\right\rangle$ and mean streamwise thermal diffusion $\tilde{a}^{2}\langle\Theta\rangle / P e$ are negligible, as reported by Piller [7] for $\operatorname{Pr}=0.71$. At $\operatorname{Pr}=0.71$ a slightly higher (in magnitude) contribution due to the radial 40 turbulent heat flux for IT, compared to MBC, is compensated by the higher (in magnitude) contribution due to the mean advective heat flux such that the 

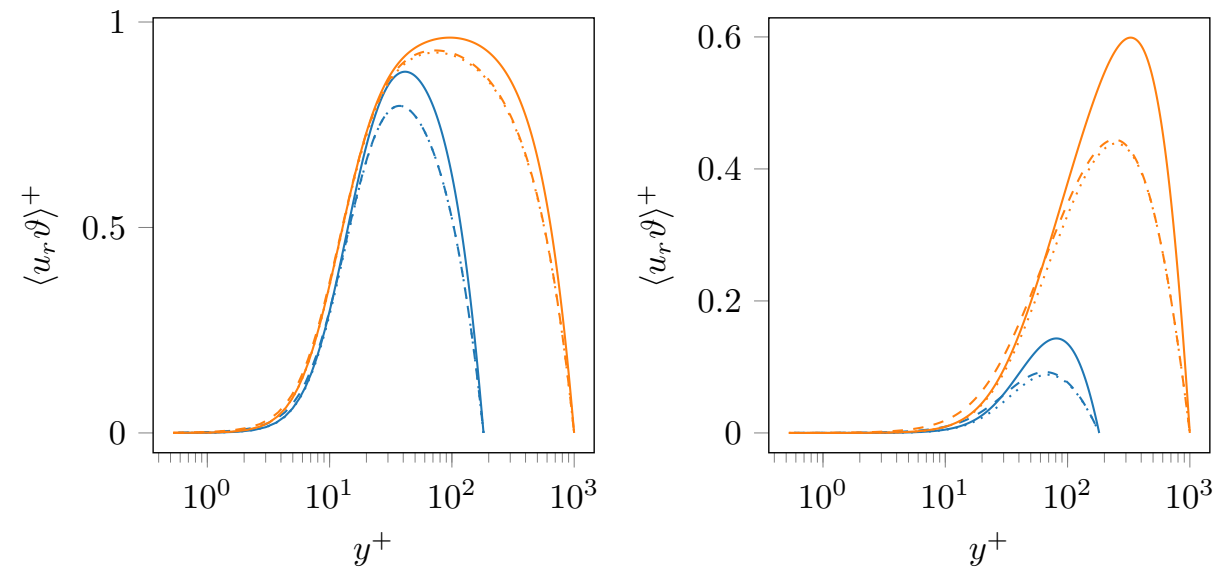

Figure 11: Variation of radial turbulent heat flux with TBC and $\operatorname{Re}_{b}$ for (left) $\operatorname{Pr}=0.71$ and (right) $\operatorname{Pr}=0.025$. Line styles and colors as in Table 3

temperature profiles are barely distinguishable. At $\operatorname{Pr}=0.025$ however, the higher (in magnitude) contribution due to the radial turbulent heat flux for IT, compared to $\mathrm{MBC}$, is overcompensated by the contribution due to the mean advective heat flux, yielding the observed higher temperature and consequently lower Nusselt number compared to MBC/IF. Note that since for IT the mean advective heat flux directly depends on the temperature, these two are interrelated such that a higher temperature yields a higher mean advective heat flux and vice versa. The sum of the contributions shown in Fig. 10 is the mean temperature $\langle\Theta\rangle$, which clearly shows the flat (turbulent) profile at $\operatorname{Pr}=0.71$ and the more rounded (laminar-like) profile at $\operatorname{Pr}=0.025$.

Fig. 11 shows the effect of the TBC and Reynolds number on the radial turbulent heat flux. For both Prandtl numbers, IT yields higher radial turbulent 485 heat fluxes whereas MBC and IF nearly collapse. Therefore, the Nusselt number contributions due to the radial turbulent heat flux for $\mathrm{MBC}$ and $\mathrm{IF}$ are very similar, as discussed previously. 

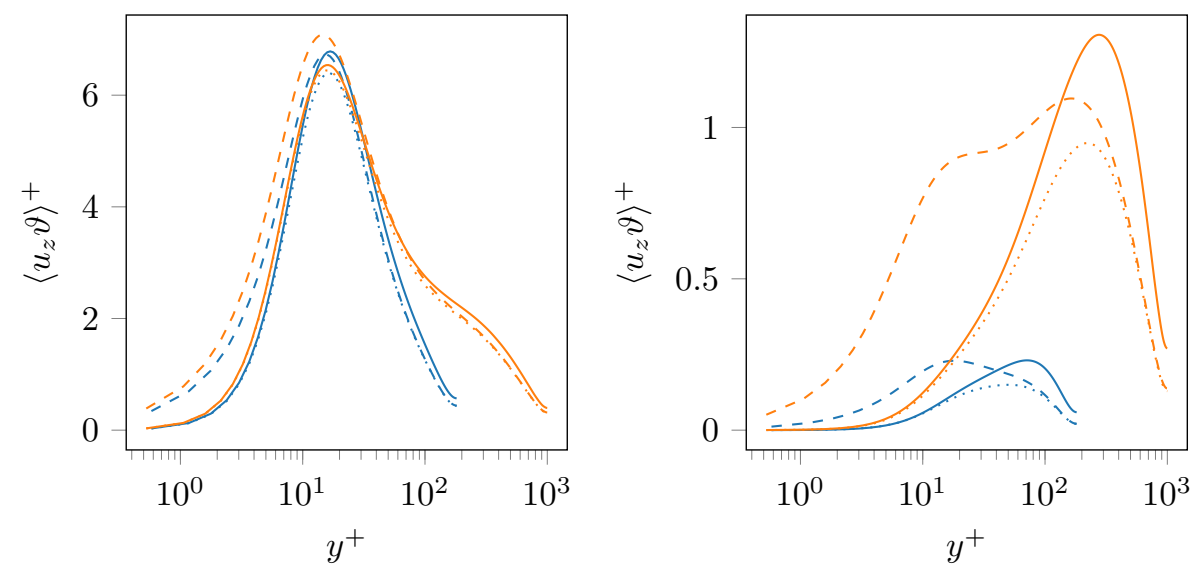

Figure 12: Variation of streamwise turbulent heat flux with TBC and $R e_{b}$ for (left) $\operatorname{Pr}=0.71$ and (right) $\operatorname{Pr}=0.025$. Line styles and colors as in Table 3

In contrast to IF, MBC/IT suppress thermal fluctuations at the wall because of the imposed Dirichlet boundary condition. Therefore, the near-wall behavior of the turbulent heat fluxes for IF is different than for MBC/IT. As given by Kong et al. [5], a Taylor series expansion for the radial and streamwise turbulent heat fluxes yields

$$
\begin{aligned}
& \left\langle\tilde{u}_{r} \vartheta\right\rangle= \begin{cases}c_{3} \tilde{y}^{3}+\ldots & \mathrm{IT}, \mathrm{MBC} \\
c_{4} \tilde{y}^{2}+\ldots & \mathrm{IF}\end{cases} \\
& \left\langle\tilde{u}_{z} \vartheta\right\rangle= \begin{cases}c_{1} \tilde{y}^{2}+\ldots & \mathrm{IT}, \mathrm{MBC} \\
c_{2} \tilde{y}+\ldots & \mathrm{IF}\end{cases}
\end{aligned}
$$

The near-wall asymptotic behavior is hardly distinguishable for the radial turbulent heat flux (quadratic vs. cubic, Eq. (14)), while for the streamwise turbulent heat flux the differences are more pronounced (linear vs. quadratic, Eq. (15)).

The streamwise turbulent heat flux is shown in Fig. 12, and different behaviors in the near-wall region are observed according to Eq. 15 and as previously discussed for the radial counterpart. The maximum streamwise turbulent heat flux for $\operatorname{Pr}=0.71$ remains at $y^{+} \approx 20$, independently of the Reynolds num- 

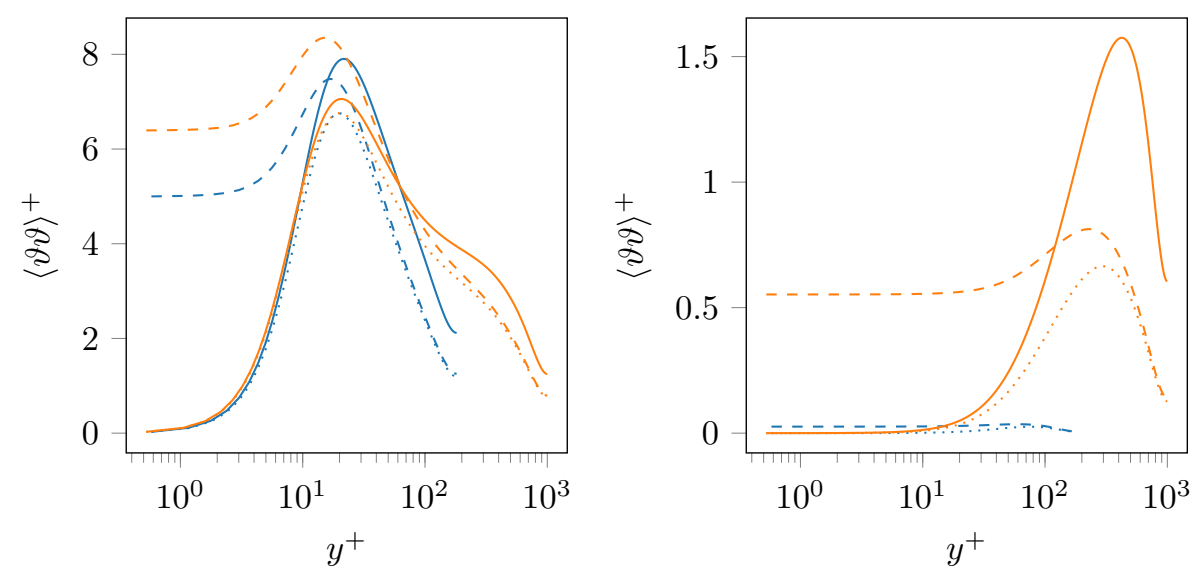

Figure 13: Variation of temperature variance with $\mathrm{TBC}$ and $\operatorname{Re}_{b}$ for (left) $\operatorname{Pr}=0.71$ and (right) $\operatorname{Pr}=0.025$. Line styles and colors as in Table 3

Differences in the near-wall behavior, arising from the TBC, will be further discussed for the temperature variance shown in Fig. 13 . The temperature variance of MBC collapses with IT close to the wall and with IF in the center of the pipe, portraying precisely the mixed behavior of MBC. The temperature 15 variation for IF at the wall is non-negligible and exhibits a constant region approximately up to $y^{+}=2$ for $\operatorname{Pr}=0.71$ and $y^{+}=30$ for $\operatorname{Pr}=0.025$. As 
for the turbulent heat fluxes, the inner-scaled temperature variance increases monotonically with increasing Reynolds number for $\operatorname{Pr}=0.025$. For $\operatorname{Pr}=0.71$, the temperature variance at the wall increases only slightly for IF with increasing Reynolds number. For MBC, the near wall behavior including the peak at $y^{+} \approx 20$ appears to be independent of the Reynolds number. The same holds for IT when $R e_{b} \geq 11700$.

\subsection{Spectral Analysis}

Streamwise power spectral densities (PSDs) of the thermal fluctuations, computed as the product of the Fourier coefficients of temperature fluctuation with their complex conjugate (i.e. $E=\hat{\vartheta} \hat{\vartheta}^{*}$ ) are evaluated for the different TBCs and Reynolds numbers. Contour lines of the premultiplied PSDs, i.e. $k_{z} \cdot E$, as a function of the wall-normal location and the streamwise wavelength $\lambda_{z}=2 \pi / k_{z}$, scaled with their respective maximum value, are shown in Fig. 14 For $\operatorname{Pr}=0.71$ the contour lines at $50 \%$ of the maximum include much smaller structures than for $\operatorname{Pr}=0.025$ because of the high thermal diffusivity at low Prandtl numbers. For $\mathrm{Pr}=0.025$ the inner-scaled streamwise wavelength of thermal structures increases at higher Reynolds numbers as observed by a shift of the contour lines and maximum values towards larger wavelengths. For instance, the maximum values for MBC reside at $\left(\lambda_{z}^{+}, y^{+}\right)=(560,80),(640,140)$, $(1060,220),(1790,420)$ for increasing Reynolds numbers. Note that as discussed previously in Section 2.1 the low Prandtl number case of IT at $R e_{b}=5300$ is excluded. For $\operatorname{Pr}=0.71$ the effect of the Reynolds number on the location of the maximum and on the contour lines is less pronounced so that the maximum ${ }_{540}$ values for $\mathrm{MBC}$ reside at $\left(\lambda_{z}^{+}, y^{+}\right)=(640,19),(560,19),(490,19),(730,17)$. One exception is the maximum value of IF at $R e_{b}=5300$ that is found at a larger wavelength and closer to the wall.

While IT and MBC show very similar PSDs at their respective Reynolds numbers, IF is characterized by temperature fluctuations occurring at the wall.

545 Hence, thermal scales of considerable streamwise wavelength are observed in the spectrum down to the wall, highlighting the difference between IF and the 

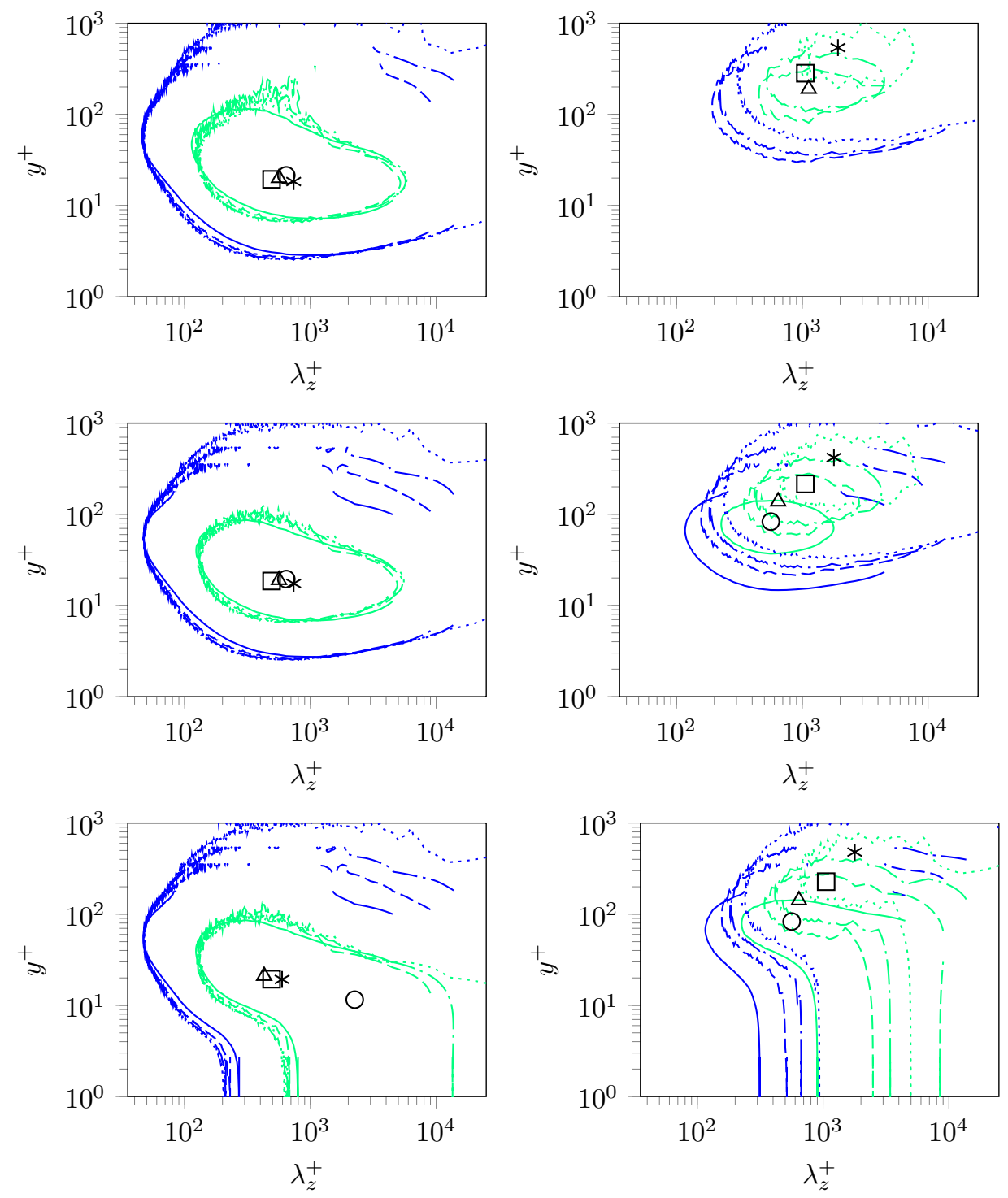

Figure 14: Streamwise premultiplied power spectral densities of thermal fluctuations at $R e_{b}=$ 5300: - - $R e_{b}=11700:---, R e_{b}=19000: \quad \cdots, R e_{b}=37700: \quad \cdots \cdots$ for (left panels) $\operatorname{Pr}=0.71$ and (right panels) $P r=0.025$. From top to bottom rows: IT, MBC, IF. Contour lines represent $50 \%$ : - and $10 \%$ : - of the maximum value, which is located at the markers $R e_{b}=5300: \circ, R e_{b}=11700: \Delta, R e_{b}=19000: \square, R e_{b}=37700: *$. Line styles as in Table 3 
other two TBCs.

\section{Conclusions}

Three types of thermal boundary conditions, i.e. isothermal, mixed-type and isoflux, are analyzed in a fully-developed turbulent forced convection pipe flow. The influence of the TBC on thermal statistics is assessed for bulk Reynolds numbers up to $R e_{b}=37700$ and Prandtl numbers of $\operatorname{Pr}=0.71, \operatorname{Pr}=0.025$ by means of DNS (for $R e_{b}=5300$ ) and well-resolved LES. For $P r=0.71$ the TBC barely affects the Nusselt number while for $\operatorname{Pr}=0.025$, the Nusselt number for IT is $\approx 20 \%$ lower than for IF/MBC analogous to laminar fully-developed forced convection. This highlights the different heat transfer mechanism for low Prandtl number fluids, where the molecular conduction plays an important role even for turbulent convection at moderately high Reynolds numbers. Thermal one-point statistics are dependent on the TBC, especially for low Prandtl numbers. Due to the Dirichlet boundary condition of IT and MBC, temperature fluctuations at the wall are suppressed, leading to different near-wall behaviors of the turbulent heat fluxes compared to IF. For the same reason the premultiplied power spectral densities of IF exhibit thermal structures at the wall, which are absent for IT and MBC.

Depending on the Prandtl number and on which statistics are to be evaluated, the appropriate TBC is to be chosen. For $\operatorname{Pr} \approx 1$ all three types give similar results except for the temperature variance at the wall. Therefore, MBC is recommended due to its simplicity in implementation. On the other hand, for $\operatorname{Pr} \ll 1$, differences between the TBCs are more pronounced. If true isothermal boundary conditions are to be simulated for a low-Prandtl-number fluid, even though difficult to realize in practice, IT should be used since MBC gives significantly different results. For a constant wall heat flux condition, first-order statistics between MBC and IF agree such that MBC is recommended. Previous literature studies on conjugate heat transfer show maximum values of temperature fluctuations occuring for IF [45], representing thus the most con- 
servative boundary condition for their estimation. The recommended TBC for low-Prandtl-number heat transfer fluids in CSP plants is IF for two reasons: First, the focused solar radiation imposes a wall heat flux boundary condition on the receiver; second, the thermal fluctuations of the wall temperature, which are only reproduced by IF, should be taken into account when designing the receiver.

The presented results of first- and second-order statistics together with the Nusselt number and its contributions are hosted in the open access repository KITopen [43]. Due to the limited numerical (and experimental) data available of heat transfer in liquid metals, this database can serve as reference data for testing and improving Reynolds-averaged Navier-Stokes (RANS) models. Individual contributions to the budget of the temperature variance and budgets of the turbulent heat fluxes in radial and streamwise direction, not discussed in the present article but important for modelling, are also available in the database.

\section{Acknowledgements}

The simulations were performed on the national supercomputer Cray XC40 at the High Performance Computing Center Stuttgart (HLRS) under the grant number ctbctpf/44106. This work was performed with the help of the Large Scale Data Facility at the Karlsruhe Institute of Technology funded by the Ministry of Science, Research and the Arts Baden-Württemberg and by the Federal Ministry of Education and Research. Support from Karlsruhe House of Young Scientists (KHYS) in form of a research travel grant for SS is appreciated.

\section{A. Budget Equations}

\section{A.1. Turbulent Kinetic Energy}

The budget of the turbulent kinetic energy $k=1 / 2\left\langle u_{i} u_{i}\right\rangle$ is given as

$$
\frac{\bar{D} k}{\bar{D} t}=P^{k}+\Pi^{k}-\tilde{\epsilon}^{k}+D^{k}+T^{k}
$$


where the individual terms are the production $P^{k}$, pressure-related diffusion $\Pi^{k}$, pseudo-dissipation $\tilde{\epsilon}^{k}$, viscous diffusion $D^{k}$ and turbulent velocity related diffusion $T^{k}$, defined as (cf. [26])

$$
\begin{aligned}
P^{k} & =-\left\langle u_{i} u_{k}\right\rangle \frac{\partial\left\langle U_{i}\right\rangle}{\partial x_{k}}, & \Pi^{k} & =-\frac{1}{\rho}\left\langle u_{i} \frac{\partial p^{\prime}}{\partial x_{i}}\right\rangle, \\
D^{k} & =\frac{\nu}{2} \frac{\partial^{2}\left\langle u_{i} u_{i}\right\rangle}{\partial x_{k} \partial x_{k}}, & T^{k} & =-\frac{1}{2} \frac{\partial\left\langle u_{i} u_{i} u_{k}\right\rangle}{\partial x_{k}}
\end{aligned}
$$

Note that the pseudo-dissipation is defined to be positive, as given in Pope [46], whereas Ref. 26] defines it to be negative, and the pressure is decomposed into mean and fluctuating part as $p=\langle p\rangle+p^{\prime}$, while velocity and thermal field are decomposed as in Eq. 10 .

\section{A.2. Temperature Variance and Turbulent Heat Fluxes}

Additionally, the temperature variance and turbulent heat flux budgets are presented for completeness, since the individual contributions are also contained in the database. The budgets for the temperature variance with $k^{\vartheta}=1 / 2\langle\vartheta \vartheta\rangle$ and turbulent heat fluxes $\left\langle u_{i} \vartheta\right\rangle$ are given by

$$
\begin{aligned}
\frac{\bar{D} k^{\vartheta}}{\bar{D} t} & =\left(\mathrm{P}^{\vartheta}-\epsilon^{\vartheta}+\mathrm{MD}^{\vartheta}+\mathrm{TD}^{\vartheta}+\mathrm{S}^{\vartheta}\right) \\
\frac{\bar{D}\left\langle u_{i} \vartheta\right\rangle}{\bar{D} t} & =\mathrm{P}_{i \Theta}+\mathrm{TPG}_{i \Theta}+\mathrm{TD}_{i \Theta}+\epsilon_{i \Theta}+\mathrm{MD}_{i \Theta}+\mathrm{S}_{i \theta}
\end{aligned}
$$

where the individual terms are the production $\mathrm{P}^{\vartheta}$, the dissipation $\epsilon^{\vartheta}$, the molecular diffusion $\mathrm{MD}^{\vartheta}$ and the turbulent diffusion $\mathrm{TD}^{\vartheta}$ of the temperature variance, defined as (cf.[47])

$$
\begin{aligned}
\mathrm{P}^{\vartheta} & =-\left\langle u_{i} \vartheta\right\rangle \frac{\partial\langle\Theta\rangle}{\partial x_{i}}, & \epsilon^{\vartheta} & =-\alpha\left\langle\frac{\partial \vartheta}{\partial x_{i}} \frac{\partial \vartheta}{\partial x_{i}}\right\rangle \\
\mathrm{MD}^{\vartheta} & =\frac{\alpha}{2} \frac{\partial^{2}\langle\vartheta \vartheta\rangle}{\partial x_{i} \partial x_{i}}, & \mathrm{TD}^{\vartheta} & =-\frac{1}{2} \frac{\partial\left\langle u_{i} \vartheta \vartheta\right\rangle}{\partial x_{i}}
\end{aligned}
$$

and the production $\mathrm{P}_{i \Theta}$, temperature-pressure gradient $\mathrm{TPG}_{i \Theta}$, turbulent diffusion $\mathrm{TD}_{i \Theta}$, dissipation $\epsilon_{i \Theta}$ and molecular diffusion $\mathrm{MD}_{i \Theta}$ of turbulent heat 
fluxes, defined as (cf.[47])

$$
\begin{aligned}
\mathrm{P}_{i \Theta} & =-\left\langle u_{i} u_{k}\right\rangle \frac{\partial\langle\Theta\rangle}{\partial x_{k}}-\left\langle u_{k} \vartheta\right\rangle \frac{\partial\left\langle U_{i}\right\rangle}{\partial x_{k}}, & \mathrm{TPG}_{i \Theta} & =-\frac{1}{\rho}\left\langle\vartheta \frac{\partial p^{\prime}}{\partial x_{i}}\right\rangle \\
\mathrm{TD}_{i \Theta} & =-\frac{\partial\left\langle\vartheta u_{i} u_{k}\right\rangle}{\partial x_{k}}, & \epsilon_{i \Theta} & =-\alpha(1+\operatorname{Pr})\left\langle\frac{\partial \vartheta}{\partial x_{k}} \frac{\partial u_{i}}{\partial x_{k}}\right\rangle
\end{aligned}
$$

$\operatorname{MD}_{i \Theta}=\frac{\partial}{\partial x_{k}}\left(\alpha\left\langle u_{i} \frac{\partial \vartheta}{\partial x_{k}}\right\rangle+\alpha \operatorname{Pr}\left\langle\vartheta \frac{\partial u_{i}}{\partial x_{k}}\right\rangle\right)$

Note that the dissipation of temperature variance and of the turbulent heat

fluxes is defined with a negative sign conforming to Kasagi and Ohtsubo [47.

The source term contributions for the temperature variance and turbulent heat fluxes depend on the type of thermal boundary condition 7.

$$
\begin{aligned}
\mathrm{S}^{\vartheta} & = \begin{cases}4\left\langle u_{z} \vartheta\right\rangle / D & \text { MBC, IF } \\
a\left(\left\langle U_{z}\right\rangle\langle\vartheta \vartheta\rangle+\langle\Theta\rangle\left\langle u_{z} \vartheta\right\rangle+\left\langle u_{z} \vartheta \vartheta\right\rangle-\alpha \frac{\partial\langle\vartheta \vartheta\rangle}{\partial z}+\alpha a\langle\vartheta \vartheta\rangle\right) & \mathrm{IT}\end{cases} \\
\mathrm{S}_{i \Theta} & = \begin{cases}4\left\langle u_{z} u_{i}\right\rangle / D & \text { MBC, IF } \\
a\left(\left\langle U_{z}\right\rangle\left\langle\vartheta u_{i}\right\rangle+\langle\Theta\rangle\left\langle u_{z} u_{i}\right\rangle+\left\langle u_{z} u_{i} \vartheta\right\rangle-\alpha 2\left\langle\frac{\partial \vartheta}{\partial z} u_{i}\right\rangle+\alpha a\left\langle\vartheta u_{i}\right\rangle\right) & \mathrm{IT}\end{cases}
\end{aligned}
$$

Note that the budgets for temperature variance and turbulent heat fluxes are given in dimensional units whereas Kasagi and Ohtsubo [47 state them in viscous units.

\section{B. Nusselt Number Decomposition for MBC/IF}

The herein presented Nusselt number decomposition closely follows the derivation given by Fukagata et al. 41] for MBC in the channel flow. Note that when defining the bulk temperature as in Eq. (4), an additional contribution (not present in Ref. 41] but in Ref. [42]) due to the streamwise turbulent heat flux emerges. In order to comply with the nomenclature of Ref. 41], the nondimensionalization and how to denote (non)-dimensional variables is adopted 
from Ref. 41] and thus different from the remaining part of this article. The final form of the derived decomposition is transformed into Eq. 11 using the non-dimensionalization as given in Section 2

The following derivation is for MBC and IF. Unfortunately, for the IT boundary condition, such a decomposition into laminar and turbulent contributions was not found due to the more complex source term in the temperature equation, which directly depends on the temperature.

We start our derivation with the transport equation of the temperature $T^{*}$ in dimensional form

$$
\frac{\partial T^{*}}{\partial t^{*}}+\nabla^{*} \cdot\left(\mathbf{U}^{*} T^{*}\right)=\alpha^{*} \nabla^{* 2} T^{*}
$$

Introducing the Reynolds decomposition for temperature and velocity as $T^{*}=$ $\bar{T}^{*}+T^{*^{\prime}}$ and $\mathbf{U}^{*}=\overline{\mathbf{U}}_{i}^{*}+\mathbf{u}^{*^{\prime}}$ and applying the averaging operator on Eq. B.1 yields the RANS equation for temperature in a pipe flow.

$$
\frac{\partial \bar{T}^{*}}{\partial t^{*}}+\nabla^{*} \cdot\left(\overline{\mathbf{U}} \bar{T}^{*}\right)=\alpha^{*} \nabla^{* 2} \bar{T}^{*}-\nabla^{*} \cdot\left(\overline{\mathbf{u}^{*^{\prime}} T^{*^{\prime}}}\right)
$$

In a steady-state, fully-developed pipe flow with homogeneous thermal boundary conditions $\partial(\cdot) / \partial t^{*}=0, \partial(\cdot) / \partial \varphi=0, U_{r}^{*}=U_{\varphi}^{*}=0$ and all derivatives in streamwise direction vanish except for $\partial \bar{T}^{*} / \partial z^{*}$ as the temperature varies (linearly [19]) in the streamwise direction. The remaining terms (now written in index notation for a cylindrical coordinate system) are

$$
0=-\frac{1}{r^{*}} \frac{\partial}{\partial r^{*}}\left(r^{*} \overline{u_{r}^{* \prime} T^{*^{\prime}}}\right)+\alpha^{*}\left[\frac{1}{r^{*}} \frac{\partial}{\partial r^{*}}\left(r^{*} \frac{\partial \bar{T}^{*}}{\partial r^{*}}\right)+\frac{\partial^{2} \bar{T}^{*}}{\partial z^{* 2}}\right]-\frac{\partial \bar{U}_{z}^{*} \bar{T}^{*}}{\partial z^{*}}
$$

$r^{*}, \varphi$ and $z^{*}$ denote the radial, azimuthal and streamwise direction respectively. Following the non-dimensionalization of Fukagata et al. [41, we scale space by the radius of the pipe $\delta^{*}$, velocity by twice the bulk velocity $2 U_{b}^{*}$ and the time by $2 U_{b}^{*} / \delta^{*}$. Note that this non-dimensionalization is different from the one given in Section 2 and is only employed for the Nusselt number decomposition to comply 
with Ref. 41. The temperature is expressed as

$$
\begin{aligned}
\Theta & =\frac{\bar{T}_{w}^{*}-T^{*}}{\Delta T_{z}^{*}} \\
\Delta T_{z}^{*} & =\frac{q_{w}^{*}}{\rho^{*} c_{p}^{*}\left(2 U_{b}^{*}\right)}=\frac{\delta^{*}}{4} \frac{\mathrm{d} \bar{T}_{w}^{*}}{\mathrm{~d} z^{*}}=\frac{\delta^{*}}{4} \frac{\mathrm{d} \bar{T}_{b}^{*}}{\mathrm{~d} z^{*}}
\end{aligned}
$$

where the wall heat flux is $q_{w}^{*}$ and the relation between the reference temperature $\Delta T_{z}^{*}$ and the gradient of bulk (or wall) temperature can be found by an integral energy balance of a heated section of length $\mathrm{d} z$. The temperature equation in non-dimensional form, omitting the superscript $(\cdot)^{*}$, thus becomes

$$
0=-\frac{1}{r} \frac{\partial}{\partial r}\left(r \overline{u_{r}^{\prime} \Theta^{\prime}}\right)+\frac{1}{R e_{b} \operatorname{Pr}}\left[\frac{1}{r} \frac{\partial}{\partial r}\left(r \frac{\partial \bar{\Theta}}{\partial r}\right)\right]+4 \bar{U}_{z}
$$

The last term is a result of the (linear) variation of the temperature and acts as a source term such that $\partial \bar{\Theta} / \partial z=0$. The product of bulk Reynolds number $R e_{b}=2 U_{b}^{*} \delta^{*} / \nu *$, with the kinematic viscosity $\nu^{*}$ and the Prandtl number $\operatorname{Pr}=\nu^{*} / \alpha^{*}$ is the Péclet number $P e=2 U_{b}^{*} \delta^{*} / \alpha^{*}$. The thermal boundary conditions for the mixed type boundary condition (MBC) and ideal isoflux (IF) are respectively

$$
\begin{aligned}
& \left.\Theta\right|_{w}=\Theta(r=1)=0 \\
& \frac{\partial \Theta}{\partial r}=-\frac{\partial \Theta}{\partial y}=-P e
\end{aligned}
$$

In order to relate the Nusselt number and the bulk temperature, first we integrate $\int_{0}^{1}$ B.6 $r \mathrm{dr}$ to find

$$
\left.\frac{\mathrm{d} \bar{\Theta}}{\mathrm{dr}}\right|_{w}=-\left.\frac{\mathrm{d} \bar{\Theta}}{\mathrm{dy}}\right|_{w}=-P e
$$

such that the Nusselt number can be expressed as

$$
\begin{aligned}
N u & =\frac{q_{w}^{*} D^{*}}{\left(\bar{T}_{w}^{*}-\bar{T}_{b}^{*}\right) \lambda^{*}}=\frac{-\left.\lambda^{*} \frac{\partial \bar{T}^{*}}{\partial y^{*}}\right|_{w} D^{*}}{\left(\bar{T}_{w}^{*}-\bar{T}_{b}^{*}\right) \lambda^{*}} \\
& =\left.\frac{\Delta T_{z}^{*}}{\left(\bar{T}_{w}^{*}-\bar{T}_{b}^{*}\right)} \frac{\mathrm{d} \bar{\Theta}}{\mathrm{dy}}\right|_{w} \frac{D^{*}}{\delta^{*}}=\frac{2 P e}{\Theta_{b}}
\end{aligned}
$$

The dimensionless bulk temperature is

$$
\bar{\Theta}_{b}=\frac{\int_{A^{*}} \overline{U_{z}^{*} \Theta} \mathrm{dA}^{*}}{\int_{A^{*}} \bar{U}_{z}^{*} \mathrm{dA} A^{*}}=\frac{\bar{T}_{w}^{*}-\bar{T}_{b}^{*}}{\Delta T_{z}^{*}}
$$


670 Next, we apply a triple integral on Eq. B.6. $\int_{1}^{0}\left(\int_{1}^{r}\left(\int_{1}^{r} \eta \mathrm{d} \eta\right) 1 / \eta^{2} \eta \mathrm{d} \eta\right) \bar{U}_{z} r d r$, reported here step by step. The first integral is $\int_{1}^{r}(\mathrm{~B} .6) \eta \mathrm{d} \eta$. Note that we integrate from the wall $r=1$ to the radial location $r$ and that we change the variable in the integrand from $r$ to $\eta$ because the upper limit of the integral is already given by $r$.

$$
\begin{aligned}
0 & =\int_{1}^{r}\left[-\frac{\partial}{\partial \eta}\left(\eta \overline{u_{r}^{\prime} \Theta^{\prime}}\right)+\frac{1}{P e}\left(\frac{\partial}{\partial \eta}\left(\eta \frac{\partial \bar{\Theta}}{\partial \eta}\right)\right)+4 \bar{U}_{z}\right] \eta \mathrm{d} \eta \\
& =-r \overline{u_{r}^{\prime} \Theta^{\prime}}+\frac{1}{P e} r \frac{\mathrm{d} \bar{\Theta}}{\mathrm{dr}}+1+\Phi
\end{aligned}
$$

where we have used the fact that the turbulent heat flux vanishes at the wall, substituted the gradient of $\Theta$ at the wall by Eq. (B.9) and introduced the fractional flow rate analogously to Fukagata et al. 41]

$$
\Phi=4 \int_{1}^{r} \bar{U}_{z} \eta \mathrm{d} \eta
$$

The lower and upper limits have been chosen such that we can apply known relations (or facts) at the wall. Switching upper and lower limits is obviously the same but with changed signs. Eq. B.14 gives us a balance of the radial turbulent heat flux. The second integral should give us a balance of the mean non-dimensional temperature by integrating $\int_{1}^{r}$ B.14 $1 / \eta^{2} \eta \mathrm{d} \eta$.

$$
\begin{aligned}
0 & =\int_{1}^{r}\left[-\overline{u_{r}^{\prime} \Theta^{\prime}}+\frac{1}{P e} \frac{\mathrm{d} \bar{\Theta}}{\mathrm{d} \eta}+\frac{1}{\eta}(1+\Phi)\right] \mathrm{d} \eta \\
& =-\int_{1}^{r} \overline{u_{r}^{\prime} \Theta^{\prime}} \mathrm{d} \eta+\frac{1}{P e} \bar{\Theta}+\int_{1}^{r} \frac{1}{\eta}(1+\Phi) \mathrm{d} \eta
\end{aligned}
$$

where we have used the boundary condition, Eq. (B.7). Note that the mean non-dimensional temperature, evaluated at the wall, has to vanish for MBC due to the boundary condition, Eq. B.7), but also for IF due to the definition of $\Theta$. The third integral should give us a balance of the Nusselt number. Therefore, we have to construct it such that we find the non-dimensional bulk temperature, which is related to the Nusselt number by Eq. (B.11), by integrating 
$\int_{1}^{0}$ B.17 $\bar{U}_{z} r \mathrm{dr}$

$$
\begin{aligned}
0 & =-\int_{1}^{0} \int_{1}^{r} \overline{u_{r}^{\prime} \Theta^{\prime}} \mathrm{d} \eta \bar{U}_{z} r \mathrm{dr}+\frac{1}{P e} \int_{1}^{0} \bar{\Theta} \bar{U}_{z} r \mathrm{dr}+\int_{1}^{0} \int_{1}^{r} \frac{1}{\eta}(1+\Phi) \mathrm{d} \eta \bar{U}_{z} r \mathrm{~d} \mathrm{r} \\
\frac{1}{N u} & =\frac{1}{2} \int_{1}^{0}(1+\Phi) \overline{u_{r}^{\prime} \Theta^{\prime}} \mathrm{dr}-\frac{1}{2} \int_{1}^{0} \frac{1}{r}(1+\Phi)^{2} \mathrm{dr}+\frac{2}{P e} \int_{0}^{1} \overline{u_{z}^{\prime} \Theta^{\prime}} r \mathrm{dr}
\end{aligned}
$$

690

studies [48, 49]. The laminar velocity profile is

$$
\bar{U}_{L}=1-r^{2}
$$

such that the laminar fractional flow rate can be evaluated to

$$
\Phi_{L}=2\left(r^{2}-\frac{r^{4}}{2}-\frac{1}{2}\right)
$$

To find an expression for the turbulent velocity profile, the complete velocity profile, taken from the derivation of the friction coefficient $C_{f}$ for a fully-developed pipe flow 40, by taking the balance equation for the mean velocity profile (after 
the second of the three integrals) and replacing $C_{f}$, has to be known

$$
\begin{aligned}
C_{f} & =\frac{16}{R e_{b}}+16 \int_{0}^{1} 2 r \overline{u_{r}^{\prime} u_{z}^{\prime}} r \mathrm{dr} \\
\bar{U}_{z} & =\operatorname{Re}_{b}\left[\int_{1}^{r} \overline{u_{r}^{\prime} u_{z}^{\prime}} \mathrm{d} \eta-\frac{C_{f}}{16}\left(r^{2}-1\right)\right] \\
\bar{U}_{z} & =\operatorname{Re}_{b} \int_{1}^{r} \overline{u_{r}^{\prime} u_{z}^{\prime}} \mathrm{d} \eta-\left(r^{2}-1\right)\left[1+R e_{b} \int_{0}^{1} 2 r \overline{u_{r}^{\prime} u_{z}^{\prime}} r \mathrm{dr}\right]
\end{aligned}
$$

such that the turbulent velocity profile is

$$
\bar{U}_{T}=\bar{U}_{z}-\bar{U}_{L}=R e_{b}\left[\left(1-r^{2}\right) \int_{0}^{1} 2 r \overline{u_{r}^{\prime} u_{z}^{\prime}} r \mathrm{dr}+\int_{1}^{r} \overline{u_{r}^{\prime} u_{z}^{\prime}} \mathrm{d} \eta\right]
$$

Finally, laminar and turbulent fractional flow rate are introduced into Eq. B.19 where the contributions containing $\Phi_{L}$ can be evaluated analytically.

$$
\begin{aligned}
\frac{1}{N u} & =\frac{11}{48}-\frac{1}{2} \int_{0}^{1}(1+\Phi) \overline{u_{r}^{\prime} \Theta^{\prime}} \mathrm{dr} \\
& -\frac{1}{2} \int_{0}^{1} \frac{1}{r}\left[\left(2 r^{4}-4 r^{2}\right) \Phi_{T}-\Phi_{T}^{2}\right] \mathrm{dr}+\frac{2}{P e} \int_{0}^{1} \overline{u_{z}^{\prime} \Theta^{\prime}} r \mathrm{dr}
\end{aligned}
$$

Hence, the Nusselt number is composed of four contributions: the laminar part $48 / 11$, the contribution due to the radial turbulent heat flux, a contribution solely due to the turbulent velocity field and a contribution due to the streamwise turbulent heat flux. The third term can be interpreted as a modification of the bulk temperature due to a different mean velocity profile as compared to the laminar one [42]. It arises from the streamwise variation of the mean axial advective heat flux $\partial \bar{U}_{z}^{*} \bar{T}^{*} / \partial z^{*}$ (c.f. eq. B.3 which is also the source of the laminar contribution.

\section{References}

\section{References}

[1] J. Kim, P. Moin, R. Moser, Turbulence statistics in fully developed channel flow at low Reynolds number, J. Fluid Mech. 177 (1987) 133-166. 
[2] N. Kasagi, Y. Tomita, A. Kuroda, Direct numerical simulation of passive scalar field in a turbulent channel flow, J. Heat Transf. 114 (1992) 598-606.

[3] H. Kawamura, H. Abe, Y. Matsuo, DNS of turbulent heat transfer in channel flow with respect to Reynolds and Prandtl number effects, Int. J. Heat Fluid Flow 20 (1999) 196 - 207. doi:http://dx.doi.org/10.1016/ S0142-727X (99) 00014-4.

[4] H. Abe, H. Kawamura, Y. Matsuo, Surface heat-flux fluctuations in a turbulent channel flow up to $R e_{\tau}=1020$ with $\operatorname{Pr}=0.025$ and 0.71 , Int. J. Heat Fluid Flow 25 (2004) 404 - 419. doi:https://doi.org/10.1016/j. ijheatfluidflow.2004.02.010.

[5] H. Kong, H. Choi, J. S. Lee, Direct numerical simulation of turbulent thermal boundary layers, Phys. Fluids 12 (2000) 2555. doi:10.1063/1. 1287912

[6] I. Tiselj, R. Bergant, B. Mavko, I. Bajsic, G. Hetsroni, DNS of turbulent heat transfer in channel flow with heat conduction in the solid wall, J. Heat Transf. 123 (2001) 849-857. doi http://dx.doi.org/10.1115/1.1389060.

[7] M. Piller, Direct numerical simulation of turbulent forced convection in a pipe, Int. J. Numer. Meth. Fl. 49 (2005) 583-602. doi:10.1002/fld.994.

[8] C. Flageul, S. Benhamadouche, É. Lamballais, D. Laurence, DNS of turbulent channel flow with conjugate heat transfer: Effect of thermal boundary conditions on the second moments and budgets, Int. J. Heat Fluid Flow 55 (2015) 34-44. doi 10.1016/j.ijheatfluidflow.2015.07.009.

[9] S. Pirozzoli, M. Bernardini, P. Orlandi, Passive scalars in turbulent channel flow at high Reynolds number, J. Fluid Mech. 788 (2016) 614-639.

[10] F. Alcántara-Ávila, S. Hoyas, M. Pérez-Quiles, DNS of thermal channel flow up to $R e_{\tau}=2000$ for medium to low Prandtl numbers, Int. J. Heat Mass 口 Tran. 127 (2018) 349-361. doi:10.1016/j .ijheatmasstransfer.2018.06. 149 . 
[11] Q. Li, P. Schlatter, L. Brandt, D. S. Henningson, DNS of a spatially developing turbulent boundary layer with passive scalar transport, Int. J. Heat Fluid Flow 30 (2009) 916-929. doi:10.1016/j.ijheatfluidflow. 2009.06 .007

[12] J. Pacio, C. Singer, T. Wetzel, R. Uhlig, Thermodynamic evaluation of liquid metals as heat transfer fluids in concentrated solar power plants, Appl. Therm. Eng. 60 (2013) 295-302. doi:http://dx.doi.org/10.1016/ j.applthermaleng.2013.07.010.

[13] R. Vinuesa, L. F. de Arévalo, M. Luna, H. Cachafeiro, Simulations and experiments of heat loss from a parabolic trough absorber tube over a range of pressures and gas compositions in the vacuum chamber, J. Renew. Sustain. Ener. 8 (2016) 023701. doi 10.1063/1.4944975 arXiv:http://dx.doi.org/10.1063/1.4944975

[14] NEK5000 v17.0, Release Date 2017/12/17. URL: https://nek5000.mcs. anl.gov, argonne National Laboratory, Illinois.

[15] A. T. Patera, A spectral element method for fluid dynamics: Laminar flow in a channel expansion, J. Comput. Phys. 54 (1984) 468 - 488. doi http: //dx.doi.org/10.1016/0021-9991(84)90128-1.

[16] M. O. Deville, P. F. Fischer, E. H. Mund, High-order methods for incompressible fluid flow, volume 9, Cambridge University Press, 2002.

[17] P. K. Kundu, I. M. Cohen, D. R. Dowling, Fluid Mechanics, 5. ed. ed., Elsevier Academic Press, Amsterdam, 2012.

[18] A. Bejan, Convection Heat Transfer, Wiley, 2013.

[19] F. P. Incropera, D. P. DeWitt, T. L. Bergmann, A. S. Lavine, Fundamentals of Heat and Mass Transfer, 6. ed. ed., Wiley, Hoboken, NJ [u.a.], 2007.

[20] B. Weigand, Analytical Methods for Heat Transfer and Fluid Flow Problems, Springer Berlin Heidelberg, 2015. doi 10.1007/978-3-662-46593-6 
[21] R. Vinuesa, L. Fick, P. Negi, O. Marin, E. Merzari, P. Schlatter, Turbulence statistics in a spectral element code: a toolbox for High-Fidelity Simulations (2017). doi:10.2172/1349052

[22] P. Schlatter, R. Örlü, Turbulent boundary layers at moderate reynolds numbers: inflow length and tripping effects, Journal of Fluid Mechanics 710 (2012) 5-34. doi $10.1017 / \mathrm{jfm} .2012 .324$.

[23] R. Vinuesa, C. Prus, P. Schlatter, H. M. Nagib, Convergence of numerical simulations of turbulent wall-bounded flows and mean cross-flow struc-

घ ture of rectangular ducts, Meccanica 51 (2016) 3025-3042. doi 10.1007/ s11012-016-0558-0.

[24] F. Lluesma-Rodríguez, S. Hoyas, M. Perez-Quiles, Influence of the computational domain on DNS of turbulent heat transfer up to $R e_{\tau}=2000$ for $\operatorname{Pr}=0.71$, Int. J. Heat Mass Tran. 122 (2018) 983 - 992. doi https: //doi.org/10.1016/j.ijheatmasstransfer.2018.02.047.

[25] L. Marocco, G. Cammi, J. Flesch, T. Wetzel, Numerical analysis of a solar tower receiver tube operated with liquid metals, Int. J. Therm. Sci. 105

口 (2016) 22 - 35. doi http://dx.doi.org/10.1016/j.ijthermalsci.2016. 02.002

[26] G. K. El Khoury, P. Schlatter, A. Noorani, P. F. Fischer, G. Brethouwer, A. V. Johansson, Direct numerical simulation of turbulent pipe flow at moderately high Reynolds numbers, Flow Turbul. Combust. 91 (2013) 475-495. doi $10.1007 /$ s10494-013-9482-8

[27] L. Marocco, Hybrid LES/DNS of turbulent forced and aided mixed convection to a liquid metal flowing in a vertical concentric annulus, Int. J. Heat Mass Tran. 121 (2018) 488 - 502. doi:https://doi.org/10.1016/j. ijheatmasstransfer.2018.01.006

[28] G. Grötzbach, Revisiting the resolution requirements for turbulence simulations in nuclear heat transfer, Nucl. Eng. Des. 241 (2011) 4379 
- 4390. doi:https://doi.org/10.1016/j.nucengdes.2010.12.027, 13th

International Topical Meeting on Nuclear Reactor Thermal Hydraulics (NURETH-13).

[29] P. Schlatter, S. Stolz, L. Kleiser, LES of transitional flows using the approximate deconvolution model, Int. J. Heat Fluid Flow 25 (2004) 549 - 558. doi:http://dx.doi.org/10.1016/j.ijheatfluidflow.2004.02.020.

[30] P. Negi, R. Vinuesa, A. Hanifi, P. Schlatter, D. Henningson, Unsteady aerodynamic effects in small-amplitude pitch oscillations of an airfoil, Int.

[34] I. Tiselj, Tracking of large-scale structures in turbulent channel with direct numerical simulation of low Prandtl number passive scalar, Phys. Fluids 830

[35] VDI Heat Atlas, Springer Berlin Heidelberg, 2010. doi 10.1007/ 978-3-540-77877-6. 
[36] J. Pacio, L. Marocco, T. Wetzel, Review of data and correlations for turbulent forced convective heat transfer of liquid metals in pipes, Heat Mass Transfer 51 (2015) 153-164. doi:10.1007/s00231-014-1392-3.

[37] V. Tricoli, Technical note Heat transfer in turbulent pipe flow revisited: similarity law for heat and momentum transport in low-Prandtlnumber fluids, Int. J. Heat Mass Tran. 42 (1999) 1535-1540. doi 10.1016/ s0017-9310(98)00258-0.

[38] E. Skupinski, J. Tortel, L. Vautrey, Determination des coefficients de convection d'un alliage sodium-potassium dans un tube circulaire, Int. J. Heat Mass Tran. 8 (1965) 937 - 951. doi:https://doi.org/10.1016/ 0017-9310(65) 90077-3.

[39] B. Lubarsky, S. J. Kaufman, Review of experimental investigations of liquid-metal heat transfer, Technical Note 3336, National Advisory Comitee for Aeronoutics, Lewis Flight Propulsion Laboratory Cleveland, Ohio, 1955.

[40] K. Fukagata, K. Iwamoto, N. Kasagi, Contribution of Reynolds stress distribution to the skin friction in wall-bounded flows, Phys. Fluids 14 (2002) L73-L76. doi:10.1063/1.1516779.

[41] K. Fukagata, K. Iwamoto, N. Kasagi, Novel turbulence control strategy for simultaneously achieving friction drag reduction and heat transfer augmentation, in: Proc. 4th Int. Symp. Turbulence and Shear Flow Phenomena, 2005, pp. 307-312.

${ }_{855}$ [42] N. Kasagi, Y. Hasegawa, K. Fukagata, K. Iwamoto, Control of turbulent transport: Less friction and more heat transfer, Journal of Heat Transfer 134 (2012) 031009. doi:10.1115/1.4005151.

[43] S. Straub, Statistics on turbulent forced convection pipe flow with different 口 thermal boundary conditions for two Prandtl numbers, 2019. doi 10.5445/ 860 IR/1000096346. 
[44] B. Kader, Temperature and concentration profiles in fully turbulent boundary layers, Int. J. Heat Mass Tran. 24 (1981) 1541 - 1544. doi https: //doi.org/10.1016/0017-9310(81)90220-9.

[45] I. Tiselj, L. Cizelj, DNS of turbulent channel flow with conjugate heat transfer at Prandtl number 0.01, Nucl. Eng. Des. 253 (2012) 153 - 160. doi:http://dx.doi.org/10.1016/j.nucengdes.2012.08.008.

[46] S. B. Pope, Turbulent Flows, 1. publ. ed., Cambridge Univ. Press, Cambridge, 2000.

[47] N. Kasagi, Y. Ohtsubo, Direct numerical simulation of low Prandtl number thermal field in a turbulent channel flow, in: F. Durst, R. Friedrich, B. E. Launder, F. W. Schmidt, U. Schumann, J. H. Whitelaw (Eds.), Turbulent Shear Flows 8, Springer Berlin Heidelberg, Berlin, Heidelberg, 1993, pp. 97-119.

[48] B. Eckhardt, S. Grossmann, D. Lohse, Torque scaling in turbulent taylor-couette flow between independently rotating cylinders, Journal of Fluid Mechanics 581 (2007) 221-250. doi 10.1017/s0022112007005629.

[49] D. Gatti, A. Cimarelli, Y. Hasegawa, B. Frohnapfel, M. Quadrio, Global energy fluxes in turbulent channels with flow control, Journal of Fluid Mechanics 857 (2018) 345-373. doi:10.1017/jfm.2018.749. 